\title{
Generation of heritable double muscle buttocks rabbits via myostatin mutation with CRISPR/Cas9 system
}

\section{Yu Zhang}

Guangxi university

\section{Yalin Zheng}

Guangxi University

Liyan Wu

Guangxi University

\section{Zhipeng Li}

Guangxi University

\section{Ning Xiao}

Guangxi University

Hongli Li

Guangxi University

\section{Lei An}

China Agricultural University

\section{Deshun Shi}

Guagnxi Unversity

Qingyou Liu ( $\nabla$ qyliu-gene@gxu.edu.cn )

Guangxi University

\section{Kuiqing Cui}

Guangxi University

\section{Research}

Keywords: rabbit, MSTN, Double muscle buttocks, Cas9, knockout

Posted Date: December 15th, 2020

DOI: https://doi.org/10.21203/rs.3.rs-125471/v1

License: (1) This work is licensed under a Creative Commons Attribution 4.0 International License. Read Full License 


\section{Abstract \\ Background}

Myostatin (MSTN) is a member of the transforming growth factor (TGF- $\beta$ ) superfamily and is considered to be a negative regulator that inhibits muscle development and regeneration, inactivity of MSTN gene may affect the development and regeneration of muscle in a few of animals.

\section{Results}

In the present study, the muscle mass negative regulator gene myostatin (MSTN) was knocked out at two novel sits in exon3, and the function of these mutation was determined in the offspring rabbits. The typical double muscle phenotype with hyperplasia and hypertrophy of muscle fiber was observed in the MSTN KO rabbits, and similar phenotype was confirmed in the $\mathrm{F} 1$ generation rabbits. The body weight of $\mathrm{MSTN}-\mathrm{KO}^{+/-}$rabbits $3708 \pm$ $43.06 \mathrm{~g}$ were significant heavier at 180 days than that of control WT rabbit $3224 \pm 48.64 \mathrm{~g}(P<0.001)$. Fourteen litters of F1 generation rabbits were produced, and the mutation of MSTN could be stably inherited in the MSTN KO rabbits.

\section{Conclusions}

Heritable double muscle buttocks rabbits via myostatin mutation with CRISPR/Cas9 system were generated, which will be valuable in meat rabbits breeding and a useful animal model for the study of human muscle development related diseases.

\section{Introduction}

Myostatin (MSTM) is a member of the transforming growth factor (TGF- $\beta$ ) superfamily and is considered to be a negative regulator that inhibits muscle development and regeneration. Like many other members of the TGF- $\beta$ family, MSTN is an extracellular hormone which signal is transmitted through activin receptors. MSTN exists in an inactive state in the skeletal muscle circulatory system and restores its activity by binding to the precursor peptide, follistatin 3 and TGF- $\beta$ binding protein[1, 2]. The high affinity binding between activated MSTN and ActRIIB can led to the signal chain transmission and then inhibit the myocyte differentiation and proliferation[3-5]. Many evidences have been found that inactivity of MSTN gene may affect the development and regeneration of muscle. Mice with MSTN gene deletion showed double muscle phenomenon and a significant increase in muscle mass[6]. It was muscle hyperplasia and hypertrophy that caused a significant increase in muscle in mice. Later, natural mutations of MSTN were found in cattles[7, 8], dogs[9], sheeps[10, 11] and human[12,13]. These natural mutations Can cause obvious double muscle trait. Efforts have been done to study the function of MSTN in muscle development of animals. The 2020 Nobel Prize in Chemistry has been awarded to Emmanuelle Charpentier and Jennifer A. Doudna[14]. Their discovery of CRISPR/Cas9 enabled scientists to cut a sequence of DNA into two pieces, allowing nature to "repair" it. During the repair process, errors will be introduced to create a different sequence of DNA, thus resulting into an edited gene. "There is enormous power in this genetic tool, which affects us all," said Claes Gustafsson, chair of the Nobel Committee 
for Chemistry. "It has not only revolutionized basic science, but also resulted in innovative crops and will lead to groundbreaking new medical treatments."Gustafsson said that, as a result, any genome can now be edited "to fix genetic damage." And now, As the most efficiency genome edit tool, CRISPR/Cas 9 system has been widely used to study the role of functional genes. Knock out of MSTN with CRISPR/Cas9 system has been processed in rabbits[15, 16], sheep[17, 18], goats[19, 20], and pigs[21, 22] in the last few years. Qian, L et al. knock out the MSTN gene of Meishan pig fetal fibroblasts by Engineered zinc-finger proteins, andprepared the MSTN-KO pig by somatic cell nuclear transfer technology firstly. Compared with wild-type Meishan pigs, the lean meat rate of Meishan pigs with MSTN knockout increased by $11.62 \%[23]$. In addition, the MSTN-KO goats obtained by the Transcription activator-like effectors successfully, and MSTN-KO goats meat production is $32 \%$ higher than wild-type goats.

Efficient TALEN-mediated myostatin gene editing in goats. BMC Dev Biol Zhang et al. Inserted the fat- 1 gene to the goat MSTN gene by CRISPR/Cas9. While overexpressing fat-1, the MSTN gene was knocked out. The results of PCR and gas chromatography analysis showed that the knockout efficiency of MSTN was as high as $25.56 \%$, and a significant increase in n-3PUFA level was detected in goat tissues. Zhang et al.[24] used CRISPR/Cas9 to insert the fat-1 gene into the goat MSTN gene, While overexpressing fat-1, the MSTN gene was knocked out, so this strategy edited both genes simultaneously. PCR and gas chromatography analysis results showed that the MSTN knockout efficiency was as high as $25.56 \%$, and a significant increase in the expression level of n-3PUFA was detected in goat tissues. Moreover, further studies found that loss activity of MSTN is also relate to the obesity, muscle atrophy and type 2 diabetes[25, 26].

It has a long tradition of rabbit meat production and consumption in France, Italy and Spain, and according to the database of FAOSTAT[27] the world per capita rabbit meat consumption in $0.242 \mathrm{~kg}$, while it is very high in many European countries, expecially $4.39 \mathrm{~kg}$ per capita in Italy. It presents the stable growth of rabbit meat consumption state from 2000 to 2013 in many European countries[28]. rabbit meat offers excellent nutritive and dietetic properties[29] and contais high-quality protein, low fat, low cholesterol, which is ideal meat of the obese and cardiovascular patients. In the present study, we designed a highly efficient new site, which is different from previous studies, and further verified that the exon 3 region can regulate the MSTN gene. At the same time, the double-muscle breech rabbits obtained in this experiment can be normally inherited to the offspring, and have stable double-muscle breech characterization.

\section{Materials And Methods}

\subsection{Animals}

In present study, all of the experiments on rabbit were performed by the Principle Guidance for the Use and Care of Laboratory Animals. Rabbits were fed regularly at Laboratory Animal Center of Guangxi University. The rabbits used for ovulation were approximately 6-8 months old and weighed between $3.5 \mathrm{~kg}$ to $4.5 \mathrm{~kg}$.

\subsection{SgRNA Design and Plasmid Construction}

A total of 8 sgRNAs targeting the MSTN sites were designed using the website (http://www.genomeengineering.org/) and named g1, g2, g3, g4, g5, g6, g7 and g8 (Fig. 1A). The two complementary DNA oligos were annealed to become double-stranded, and cloned into the pMD18-hU6-gRNA vector. Primer T76 and T78 
were used as target gene fragments amplified by the two sgRNA targeting vectors. Linearized DNA plasmids and PCR products were extracted and purified using the MEGA shortscrip ${ }^{\mathrm{TM}}$ T7 kit (Ambion, USA) and sgRNAs were generated according to the manufacturer's recommendations.

\subsection{Culture and electroporation of fibroblasts}

Primary rabbit fibroblasts were cultured in 10\% (v/v) FBS, $35 \mathrm{~mm}$ glutamine and $1 \times$ DMEM (Gibco). The plasmid was transfected into fibroblasts by electroporation at a pulse of 225 volts for 10 milliseconds. Briefly, $4 \mu \mathrm{g}$ of pCMV-T7-NLS-hSpCas9-NLS (hSpCas9) DNA plasmid and $2 \mu \mathrm{g}$ of gRNA plasmid were used to transfect $5-10 \times 10^{5}$ fibroblasts. the culture medium was changed to DMEM containing $10 \%$ FBS 24 hours later. After cultured for 4 days, the DNA was extracted and the MSTN was amplified by PCR. Sent to the company for sequencing to analyze the editing effect of the gene fragment. Optimal sgRNA was selected for the following experiment.

\subsection{Zygotes collection and Blastocyst genotyping testing}

Rabbits of estrus were mated two or three times 16-20 hours before mating, and zygotes were collected from the fallopian tubes with cell culture medium. The cell culture medium was consisted of M199 (Gibco, USA), 3\% bovine serum (Gibco, USA), $5 \mathrm{mM}$ Hepes (Sigma, USA) and $5 \mathrm{mM} \mathrm{NaHCO}_{3}$ (Sigma, USA). The zygotes were microinjected with Cas 9 protein and sgRNA and in vitro cultured to blastocyst, and the mutation was analyzed by PCR and T7El digestion of the target region.

\subsection{Generation of transgenic rabbit}

The zygotes was injected with $10 \mathrm{pL}$ CRISPR/Cas 9 mixture, containing $200 \mathrm{ng} / \mu \mathrm{L}$ Cas 9 protein (A36497, Hot Fisher, USA), $20 \mathrm{ng} / \mu \mathrm{L}$ sgRNA6 and $20 \mathrm{ng} / \mu \mathrm{L}$ sgRNA8, in the cytoplasm and the zygotes were transferred back to the oviduct of recipient rabbit. Pregnancy phenomenon was checked on 10 days after the transplantation and the cubs were born about 30 days later.

\subsection{T7 Endonuclease I (T7EI) assay}

Genome DNA of the cubs were extracted and the mutation induced by CRISPR/Cas 9 was analyzed by PCR and T7E1 assay. PCR was performed using primers flanking the target site and the product was sequenced (Shanghai Sangon Company, Shanghai, China) and digested with T7EI (NEB, USA) to characterize the mutation.

\subsection{Off-target analysis}

Potential off-target sites of the sgRNAs were evaluated using the CRISPR design tool (http://crispor.tefor.net/). The top 5 potential off-target sites of each sgRNA were selected and amplified by PCR with specific primers (Table.1), and the PCR products were analyzed by sequencing and T7E1 digestion.

\subsection{Western Blot analysis}

The gluteus Maximus tissue samples of $\mathrm{MSTN}^{+/-}$and WT rabbits (euthanized at 6 months of age) was collected, tissues were ground in liquid nitrogen, and added $2.5 \mu \mathrm{L} / \mathrm{mL}$ protease inhibitor with ice for 30 minutes. Protein concentration was determined using the Bradford method (Bio-Rad). $35 \mu \mathrm{g}$ of protein sample was subjected to a $5 \%$ 12\% separation of SDS-polyacrylamide gel. Antibodies used in this study included anti- 
MSTN polyclonal antibody (Abcam) and goat anti-rabbit IgG coupled to horseradish peroxidase (HRP) (Santa Cruz, USA). The $\beta$-actin antibody (Santa Cruz) was used as an internal control.

Table 1

Primers of off-target detection

\begin{tabular}{|c|c|c|}
\hline Primer & Sequence & Length \\
\hline POTs6-1F & CAATGGTGTGAGCCTCAAAG & \multirow[t]{2}{*}{$382 \mathrm{bp}$} \\
\hline P0Ts6-1R & AGTGGTCGTCTTCTTCATCC & \\
\hline POTs6-2F & ATGCTCCTGTGTAGTCACTG & \multirow[t]{2}{*}{$373 \mathrm{bp}$} \\
\hline POTs6-2R & GTTTTCCATGTCCAGCTCAC & \\
\hline POTs6-3F & ATCCAGGTATTAGCAACCGT & \multirow[t]{2}{*}{292 bp } \\
\hline P0Ts6-3R & TCTGTGAATGTGCATACATACA & \\
\hline POTs6-4F & TGACTACTGGCCCAAAATGT & \multirow[t]{2}{*}{$450 \mathrm{bp}$} \\
\hline P0Ts6-4R & TTCAGTCACAGAGTCGGTTT & \\
\hline POTs6-5F & GAAGGGCCACAAAGAGAAAG & \multirow[t]{2}{*}{$238 \mathrm{bp}$} \\
\hline POTs6-5R & AAGGCCTCTTCCTCCCAG & \\
\hline POTs8-1F & GGCGCTCATGATCTCTTGCT & \multirow[t]{2}{*}{$291 \mathrm{bp}$} \\
\hline POTs8-1R & СCTCACCAATGTCGATGCCT & \\
\hline POTs8-2F & AGCAGACATTCTGGCGGAAA & \multirow[t]{2}{*}{$348 \mathrm{bp}$} \\
\hline POTs8-2R & GCCAAATGCAGCCTCAGAAT & \\
\hline POTs8-3F & TCCAGAGTGCCTGCAGGTA & \multirow[t]{2}{*}{$351 \mathrm{bp}$} \\
\hline POTs8-3R & TCCAGAAGCTCAAATCTCTTGCT & \\
\hline POTs8-4F & GTTTGGACACTGCTTGCTGG & \multirow[t]{2}{*}{$291 \mathrm{bp}$} \\
\hline POTs8-4R & TCATGGTGGATGCCCTCTTG & \\
\hline POTs8-5F & CACACACATCCTCGGCTCAT & \multirow[t]{2}{*}{$352 \mathrm{bp}$} \\
\hline POTs8-5R & САТТАССТТССТСССАСССС & \\
\hline
\end{tabular}

\subsection{Weight, and sample collection and Histological analysis}

In this study, $5 \mathrm{FO} \mathrm{MSTN}{ }^{+/-}$and $5 \mathrm{WT}$ rabbits were feed at the same conditions. All rabbits were weaned at 30 days and were kept in separate cages in the same litter. Body weight was recorded every 10 days up to 200 days. At 2 months of age, rabbits were anesthetized with anesthesia with pentobarbital sodium ( $1 \mathrm{~mL} / \mathrm{kg})$. The corpses were dissected and the hearts, tongue, gluteus maximus and lateral femoral muscles of the MSTNKO and WT groups were weighed. 
The tongue and gluteus Maximus tissues of FO generation MSTNKO and WT rabbits (6 months old sacrifice) were fixed with $4 \%$ paraformaldehyde at $4{ }^{\circ} \mathrm{C}$, The increasing concentrations of dehydrated sucrose $(30 \%$ for $7 \mathrm{~h}, 40 \%$ for $7 \mathrm{~h}, 45 \%$ for $5 \mathrm{~h}$ ), freezed section at $-25^{\circ} \mathrm{C}$ for histological examination. The tissues which were sectioned into $5 \mu \mathrm{m}$ thick slices were stained with hematoxylin and eosin (H\&E) and analyzed by Fluorescence Inverted Microscope (Nikon, Japan). The integral optical density (IOD) analysis of histological sections was performed by Image-Pro Plus 6.0 software.

\subsection{Statistical analysis}

All of the data we obtained in this study were analyzed using Graphpad prism software (T test) and one-way ANOVA followed by Duncan's Multiple Comparison Tests in the SPSS 17 statistical software package.

\section{Results}

\subsection{Design and construction of sgRNA}

Eight sgRNAs targeting to the MSTN exon 1 and exon 3 were designed and cloned into the pMD18T vector, respectively (Fig. 1A). The recombinant vector was named as pMD18-hU6-gRNA and confirmed that the sgRNA has been successfully inserted into the vector by sequence analysis (Fig. 1C).

\subsection{Modification efficiency of the sgRNAs in rabbit fibroblasts}

The mutation efficiency of these designed sgRNAs was comfirmed in rabbit fibroblasts, and no mutation were generated in $\mathrm{g} 1$ to $\mathrm{g} 4$, the mutation rates of g5-g8 from 20-45\% (Fig. 2). The sgRNAs target to exon 3 is significantly better than that of exon 1 . The gene modification rate of sgRNA6 $(45 \%, 9 / 20)$ and sgRNA8 (40\%, $8 / 20$ ) were higher than that of the other gRNAs. Thus, sgRNA6 and sgRNA8 were selected in the following experiments.

\subsection{CRISPR/Cas9-mediated MSTN site-specific KNOCK-OUT in rabbit zygotes}

The CRISPR/Cas9 system containing sgRNA6 and sgRNA8 were injected into the zygotes to verify the sitespecific deletion of MSTNgene. Atotalof16 zygotes was injected and 10 of them developed to blastocyst stage in vitro (Fig. 3A). The genome editing efficiency in the blastocysts were analyzed by PCR and Sanger sequence, and results revealed that 7 of them showed geneticmutations $(70 \%, 7 / 10)$ (fig. 3B).

\subsection{Generation of MSTN knock-out rabbits}

A total of 99 cytoplasmic injected zygotes were transplanted to oviduct of 10 receptor female rabbits, and 23 pup rabbits were born after full-term gestation (Table 2). The presence of genome editing was analyzed by T7E1 digestion and Sanger sequencing analysis, and the results showed that 5 of them are MSTNknock-out rabbits (Fig. 4). Intrestingly, three $\mathrm{MSTN}^{+/-}$rabbits were all males, and two $\mathrm{MSTN}^{-/-}$rabbits were all females. The further T7E1 and sequencing analysis showed that 5 MSTN knock-out rabbit are all chimeras. 
Table 2

Generation of the MSTNKO rabbits via CRISPR/Cas9

\begin{tabular}{|c|c|c|c|c|c|c|c|}
\hline NO. & SgRNA & $\begin{array}{l}\text { gRNA/Cas9 } \\
\text { protein(ng/uL) }\end{array}$ & $\begin{array}{l}\text { Embryos } \\
\text { Injected }\end{array}$ & $\begin{array}{l}\text { Embryos } \\
\text { transferred }\end{array}$ & Pregnancy & $\begin{array}{l}\text { Pups } \\
\text { obtained } \\
\text { (\%transferred) }\end{array}$ & $\begin{array}{l}\text { MSTNKO } \\
\text { pups(\%pups) }\end{array}$ \\
\hline M02 & $\begin{array}{l}\text { Sg6 } 6+ \\
\text { Sg8 }\end{array}$ & $40 / 200$ & 14 & 14 & Yes & $3(21.4 \%)$ & $1(33.33 \%)$ \\
\hline M08 & $\begin{array}{l}\text { Sg6 }+ \\
\text { Sg8 }\end{array}$ & $40 / 200$ & 12 & 12 & Yes & $3(25 \%)$ & $1(33.33 \%)$ \\
\hline M09 & $\begin{array}{l}\text { Sg6 } 6+ \\
\text { Sg8 }\end{array}$ & $40 / 200$ & 7 & 7 & Yes & $4(57.14 \%)$ & $0(0)$ \\
\hline M10 & $\begin{array}{l}\text { Sg6 }+ \\
\text { Sg8 }\end{array}$ & $40 / 200$ & 10 & 10 & Yes & $4(40)$ & $2(50 \%)$ \\
\hline M12 & $\begin{array}{l}\text { Sg6 }+ \\
\text { Sg8 }\end{array}$ & $40 / 200$ & 9 & 8 & Yes & $4(50 \%)$ & $1(25 \%)$ \\
\hline M13 & $\begin{array}{l}\text { Sg6 }+ \\
\text { Sg8 }\end{array}$ & $40 / 200$ & 8 & 8 & Yes & $3(37.5 \%)$ & $0(0)$ \\
\hline M14 & $\begin{array}{l}\text { Sg6 }+ \\
\text { Sg8 }\end{array}$ & $40 / 200$ & 7 & 14 & Yes & $2(14.29 \%)$ & $1(50 \%)$ \\
\hline M15 & $\begin{array}{l}\text { Sg6 }+ \\
\text { Sg8 }\end{array}$ & $40 / 200$ & 9 & 7 & Yes & $4(57.14 \%)$ & $1(25 \%)$ \\
\hline M17 & $\begin{array}{l}\text { Sg6 }+ \\
\text { Sg8 }\end{array}$ & $40 / 200$ & 11 & 11 & Yes & $4(36.36 \%)$ & $2(50 \%)$ \\
\hline
\end{tabular}

\subsection{Off-target analysis of MSTN knock-out rabbits}

To detect whether off-target mutations were present in those MSTN knock-outrabbits, 4 potential offpotspersgRNA were designed using the onlineCRISPR Design Tool (http://crispor.tefor.net/). The further T7EI digestion and Sangersequencing resultsshowedthatnomutationwasdetected, which mean that noofftargetpresentin the MSTMKOrabbits(Fig.5).

\subsection{Western Blot analysis of MSTN KO rabbits}

The expressionof MSTNin muscle tissue of MSTNKO rabbit was significantly lower than that of WT rabbit based on QRT-PCR analysis $(p<0.05)$. And the further western blot analysis results showed that the expression level of MSTN protein of the mutant rabbits was lower in the muscle than wild rabbit(Fig. 6).

\subsection{Morphological analysis of MSTN KO rabbits}

The difference in muscle development of the MSKNKO rabbit was evaluated by morphological analysis and body weight. The specific data of weight gain are shown in Table 3. No significant difference of the body weight was found in the five MSTNKO rabbits with the control group within the early 90 days $(P>0.05)$. When the five MSTNKO rabbits grown to the third month, the weight of MSTNKO rabbits began to appear slightly higher than that of the control group (Fig. 7C). And MSTNKKO rabbits appeared "double muscle" phenomenon, rabbit muscle 
morphology was more obvious. At 130 days of age, the weight of five MSTN-KO rabbits showed a significant heavier than that of wild control rabbits $(P<0.05, \mathrm{n}=5)$. And the body weight of MSTN-KO rabbits $3708 \pm$ $43.06 \mathrm{~g}$ were significant heavier at 180 days than that of control WT rabbit $3224 \pm 48.64 \mathrm{~g}(P<0.001, \mathrm{n}=5)$. The gluteus of the MSTN-KO rabbits also looks bigger than WT rabbits, and the further histological analysis showed that the density and diameter of the myofiber was significantly larger in MSTN-KO rabbits (Fig. 7B).

Table 3

FO generation rabbits weight data

\begin{tabular}{|lllllllllll|}
\hline Age/Day & M2-1 & M15-2 & WT1 & WT2 & T2-3 & T7-2 & T7-3 & T2-2 & T7-1 & T7-4 \\
\hline 1 & 88 & 85.4 & 74.2 & 81.5 & 73 & 76.8 & 70.1 & 72.5 & 80.6 & 58.7 \\
\hline 10 & 165 & 206 & 240 & 218 & 200 & 335 & 320 & 220 & 365 & 160 \\
\hline 20 & 326 & 360 & 459 & 431 & 410 & 460 & 480 & 430 & 490 & 310 \\
\hline 30 & 650 & 690 & 770 & 720 & 760 & 680 & 640 & 860 & 720 & 360 \\
\hline 40 & 1090 & 1200 & 1132 & 1090 & 1250 & 1190 & 1148 & 1280 & 1300 & 1228 \\
\hline 50 & 1450 & 1690 & 1680 & 1620 & 1560 & 1330 & 1355 & 1435 & 1470 & 1250 \\
\hline 70 & 1900 & 1930 & 2130 & 1940 & 1960 & 1670 & 1800 & 2100 & 1890 & 1810 \\
\hline 80 & 2270 & 2380 & 2320 & 2400 & 2030 & 1735 & 2095 & 2240 & 2180 & 2130 \\
\hline 90 & 2810 & 2660 & 2656 & 2595 & 2385 & 2080 & 2250 & 2415 & 2285 & 2390 \\
\hline 100 & 3119 & 2980 & 3120 & 2940 & 2630 & 2275 & 2340 & 2790 & 2585 & 2820 \\
\hline 110 & 3420 & 3100 & 3160 & 3090 & 2980 & 2380 & 2460 & 2980 & 2600 & 2880 \\
\hline 120 & 3520 & 3300 & 3180 & 2980 & 2940 & 2410 & 2580 & 3200 & 2780 & 2950 \\
\hline 130 & 3490 & 3350 & 2800 & 3100 & 2920 & 2570 & 2660 & 3280 & 2880 & 3060 \\
\hline 140 & 3448 & 3670 & 2960 & 3200 & 2980 & 2630 & 2750 & 3430 & 2960 & 3130 \\
\hline 150 & 3480 & 3790 & 2840 & 3160 & 3120 & 2870 & 2930 & 3610 & 3280 & 3310 \\
\hline 160 & 3580 & 3830 & 3060 & 3340 & 3100 & 2910 & 3060 & 3690 & 3480 & 3380 \\
\hline 170 & 3530 & 3750 & 2980 & 3300 & 3270 & 3110 & 3200 & 3750 & 3650 & 3440 \\
\hline Note:The weight unit is g. & & & & & & & & \\
\hline
\end{tabular}

\subsection{Heritability of the MSTN-KO rabbits.}

To determine whether the MSTNKO could be stably transmitted to the offspring, the two female founders $M S T N^{\wedge}$ - were mated with male rabbit, but now offspring pub rabbits were produced. The threeMSTN ${ }^{+/} \mathrm{F} 0$ male rabbits were mated with wild rabbits, and live F1 offspring pub rabbits generated from all those three rabbits. T- 
cloning sequence analysis and T7E1 cleavage assay demonstrated that 28 out of 53 newborn F1 rabbits carried MSTN mutations. In these pups, the 23 were monoallelic, while 5 were biallelic MSTN-KO rabbits (Fig. 8).

\section{Discussion}

In this experiment, the Cas9 / sgRNA mixture was injected into the single-cell stage embryos by cytoplasmic injection to delete long fragments and ensure the inactivation of target gene function. The MSTNKO rabbit was successfully produced, and the results showed that the system can effectively produce knockout rabbits. The loss of the MSTN fragment could leads to muscle growth.

Although the increase in muscle mass makes MSTN-KO livestock production more attractive, However, in these MSTN mutant animals, calving difficulties are often caused by various reasons. MSTN is an important regulator of muscle cell proliferation and differentiation during muscle development. MSTN signals can be detected from the early myogenic stage of embryonic sarcomere formation to the adult skeletal muscle development stage[7, $30,31]$. The expression of MSTN was observed for the first time during the embryonic period[7, 30, 31]. The birth weight of MSTN mutant animals has been observed in sheep, cattle and goats[32, 33].Therefore, the knockout of the MSTN gene may affect muscle development in the embryonic stage. The researchers believe that during fetal development, MSTN is only expressed in skeletal muscle, thereby controlling the differentiation and proliferation of myoblasts. In our study, MSTN-KO rabbits exhibited a typical double-muscle phenotype, but there was no significant difference in body weight at first 60 days and Compared with the wild-type control group, there is almost no difference in body size at birth $(P>0.05)$. It's might beacause IGF-1 is an important positive regulator of muscle cell proliferation and differentiation in skeletal muscle[34]. Despite the inhibition of MSTN signal expression, IGF signaling will still upregulate the expression of myostatin in skeletal muscle tissue models, which also indicates that there is an inhibitory autoregulatory loop in the muscular system[35, 36].

Studies believe that in most terrestrial vertebrates, the increase in mammalian muscle production is mainly caused by muscle fiber hypertrophy, hyperplasia, or a combination thereof caused by the lack of MSTM21, 3739]. The number of muscle fibers mainly determined before birth, and the diameter of muscle fibers may expand after birth $[40,41]$. The muscle mechanisms of hypertrophy and hyperplasia may not occur simultaneously[42, 43]. But it seems that the mechanism by which MSTN mutations enhance muscle mass is different between species. In MSTN mutant mice, goats and rabbits, the increase in muscle mass is caused by muscle fiber hyperplasia and diameter hypertrophy[37, 44, 45]. However, only muscle fiber hyperplasia was observed in MSTN mutant cattle and pigs[8, 46]. The muscle mass of MSTN-deficient mice is 2-3 times than of wild-type mice. The increase in muscle mass is the result of the combined action of muscle fiber hyperplasia and hyperplasia[37]. In contrast, double-muscle cattle show muscle mass increased by $20 \%-25 \%$, and the increased muscle weight seems to be the result of muscle fiber diameter proliferation, rather than an increase in muscle number[8,39]. The phenotypic trait of muscle hypertrophy is a quantitative trait. The quantitative trait is the result of the joint action of many independent small-effect multi-genes. The effect of each gene is equal, but in some cases there is a major effect gene Gene is the main effect in this trait, and the other is micro-effect. The muscle growth inhibition is controlled by MSTN gene, and other genes are controlled by the micro-effect gene.

In addition, the MSTN-KO rabbit exhibits the muscular feature of "double mushy buttocks", and the fetus of the rabbit presents with "big tongue syndrome"[47]. In some live rabbits born, some of the MSTN-KO rabbits also have "big tongue syndrome", which causes milk sucking. Success leads to death. Most of the MSTN-KO rabbits 
show a rapid increase in muscle mass after 60 days of age, and MSTN-KO rabbits are healthy and can inherit the knocked-out gene fragments, which shows that the MSTN-KO rabbits produced by the CRISPR / Cas 9 system are suitable for studying muscle development and Related diseases.

\section{Conclusion}

In summary, we obtained MSTN-KO rabbits with double muscle typically. These MSTN-KO rabbits could be a promising tool for studying the development of livestock muscles and improving their important economic traits. Thereby improving the meat production efficiency of rabbits and promoting the development of rabbit industry. We established a CRISPR/Cas9 gene editing system suitable for the laboratory, which is more effectively applied to various model animals and large livestock animals.

\section{Abbreviations}

CRISPR: Clustered regularly interspaced short palindromic repeats

\section{Declarations}

\section{Ethics approval and consent to participate}

All experimental studies were approved by the Experimental Animal Care and Use Committee of Guangxi University (Permit code: GXU2019-029).

\section{Consent for publication}

Not applicable.

\section{Availability of data and materials}

All datasets for this study are included in the manuscript and the Supplementary Files. The datasets used or analysed during the current study available from the corresponding author on reasonable request.

\section{Competing interests}

The authors declare that there is no conflict of interest.

\section{Funding}

Present study was granted and supported by the National Natural Science Fund (Grant No. 31760648 and 31860638 ). and we are also grateful for Guangxi Natural Science Foundation (Grant No. AB16380042).

\section{Authors' contributions}

QYL and KQC designed the experiments, YZ and YLZ conceived the experiments and draft the manuscript. LYW, NX, and HLL help performed the experiments. YZ and LYW analyzed the data. ZPL , LA and DSS revised the manuscript. All authors read and approved the final manuscript.

\section{Acknowledgements}


Not applicable.

\section{References}

1. Rodino-Klapac LR, Haidet AM, Kota J, Handy C, Kaspar BK, Mendell JR. Inhibition of myostatin with emphasis on follistatin as a therapy for muscle disease. Muscle Nerve. 2009;39(3): 283-96.

2. McCoy JC, Walker RG, Murray NH, Thompson TB. Crystal structure of the wfikkn2 follistatin domain reveals insight into how it inhibits growth differentiation factor 8 (gdf8) and gdf11. The Journal of biological chemistry. 2019;294(16): 6333-43.

3. Wicik Z, Sadkowski T, Jank M, Motyl T. The transcriptomic signature of myostatin inhibitory influence on the differentiation of mouse c2c12 myoblasts. Pol J Vet Sci. 2011;14(4): 643-52.

4. Nomura T, Ueyama T, Ashihara E, Tateishi K, Asada S, Nakajima N et al. Skeletal muscle-derived progenitors capable of differentiating into cardiomyocytes proliferate through myostatin-independent tgfbeta family signaling. Biochem Biophys Res Commun. 2008;365(4): 863-9.

5. Sakamoto K, Kanematsu-Yamaki Y, Kamada Y, Oka M, Ohnishi T, Miwa M et al. Identification of ligandselective peptidic actriib-antagonists using phage display technology. Biochem Biophys Rep. 2017;11(33-9.

6. Mc Pherron A C LAML. regulation of skeletal muscle mass in mice by a new tgf-p superfamily member. Nature. 1997;387: 83-).

7. Kambadur R, Sharma M, Smith TPL, Bass JJ. Mutations in myostatin(gdf8) in double-muscled belgian blue and piedmontese cattle. Genome Research. 1997;7(9): 910-5.

8. Grobet L, Royo Martin LJ, Poncelet D, Pirottin D, Brouwers B, Riquet $J$ et al. A deletion in the bovine myostatin gene causes the double-muscled phenotype in cattle. Nature Genetics. 1997;17(1): 71-4.

9. Mosher DS, Quignon P, Bustamante CD, Sutter NB, Mellersh CS, Parker HG et al. A mutation in the myostatin gene increases muscle mass and enhances racing performance in heterozygote dogs. PLoS Genetics. 2007;3(5): e79.

10. Boman IA, Vage DI. An insertion in the coding region of the myostatin (mstn) gene affects carcass conformation and fatness in the norwegian spaelsau (ovis aries). BMC Res Notes. 2009;2(98.

11. Sahu AR, V J, R R, A R. Novel report on mutation in exon 3 of myostatin (mstn) gene in nilagiri sheep: an endangered breed of south india. Tropical animal health and production. 2019;51(7): 1817-22.

12. Saunders MA, Good JM, Lawrence EC, Ferrell RE, Li WH, Nachman MW. Human adaptive evolution at myostatin (gdf8), a regulator of muscle growth. Am J Hum Genet. 2006;79(6): 1089-97.

13. Schuelke M, Wagner KR, Stolz LE, Hubner C, Riebel T, Komen W et al. Myostatin mutation associated with gross muscle hypertrophy in a child. N Engl J Med. 2004;350(26): 2682-8.

14. Doudna JA, Charpentier E. Genome editing. The new frontier of genome engineering with crispr-cas 9. Science (New York, N.Y.). 2014;346(6213): 1258096.

15. Yan Q, Zhang Q, Yang H, Zou Q, Tang C, Fan N et al. Generation of multi-gene knockout rabbits using the cas9/grna system. Cell Regen (Lond). 2014;3(1): 12.

16. Xiao N, Li H, Shafique L, Zhao S, Su X, Zhang Y et al. A novel pale-yellow coat color of rabbits generated via mc1r mutation with crispr/cas9 system. Frontiers in genetics. 2019;10(875. 
17. Li H, Wang G, Hao Z, Zhang G, Qing Y, Liu S et al. Generation of biallelic knock-out sheep via gene-editing and somatic cell nuclear transfer. Scientific reports. 2016;6(33675.

18. Zhang Y, Wang Y, Yulin B, Tang B, Wang M, Zhang C et al. Crispr/cas9-mediated sheepmstn gene knockout and promote ssmscs differentiation. Journal of Cellular Biochemistry. 2018;120(2): 1794-806.

19. Wang X, Yu H, Lei A, Zhou J, Zeng W, Zhu H et al. Generation of gene-modified goats targeting mstn and fgf5 via zygote injection of crispr/cas9 system. Sci Rep. 2015;5(13878.

20. Zhang J, Liu J, Yang W, Cui M, Dai B, Dong Y et al. Comparison of gene editing efficiencies of crispr/cas9 and talen for generation of mstn knock-out cashmere goats. Theriogenology. 2019;132(1-11.

21. Wang K, Ouyang H, Xie Z, Yao C, Guo N, Li M et al. Efficient generation of myostatin mutations in pigs using the crispr/cas9 system. Sci Rep. 2015;5(16623.

22. Xie S, Li X, Qian L, Cai C, Xiao G, Jiang S et al. An integrated analysis of mrna and mirna in skeletal muscle from myostatin-edited meishan pigs. Genome. 2019;62(5): 305-15.

23. Qian L, Tang M, Yang J, Wang Q, Cai C, Jiang S et al. Targeted mutations in myostatin by zinc-finger nucleases result in double-muscled phenotype in meishan pigs. Sci Rep. 2015;5(14435.

24. Zhang J, Cui ML, Nie YW, Dai B, Li FR, Liu DJ et al. Crispr/cas9-mediated specific integration of fat-1 at the goat mstn locus. FEBS J. 2018;285(15): 2828-39.

25. Allen DLHD. Expression and function of myostatin in obesity, diabetes, and exercise adaptation. Med. Sci. Sports Exerc. 2011;43): 1828-35.

26. Guo TEA. Myostatin inhibition prevents diabetes and hyperphagia in a mouse model of lipodystrophy

Diabetes.61, 2414-2423).

27. Food and agriculture organization of the united nations No. 2020. p The Food and Agriculture Organization (FAO) is a specialized agency of the United Nations that leads international efforts to defeat hunger. http://www.fao.org/home/common-elements/top-navigation-content/resources/en/

28. Szendrő K, Szabó-Szentgróti E, Szigeti O. Consumers' attitude to consumption of rabbit meat in eight countries depending on the production method and its purchase form. Foods. 2020;9(5): 654.

29. Hernández, P .; Dalle Zotte, A. Influence of diet on rabbit meat quality. In Nutrition of the Rabbit, 2nd ed.; de Blas, C., Wiseman, J., Eds.; CAB International: Oxfordshire, UK, 2010; pp. 163-178

30. Grobet L, Martin LJ, Poncelet D, Pirottin D, Brouwers B, Riquet $\mathrm{J}$ et al. A deletion in the bovine myostatin gene causes the double-muscled phenotype in cattle. Nat Genet. 1997;17(1): 71-4.

31. Manceau M, Gros J, Savage K, Thome V, McPherron A, Paterson B et al. Myostatin promotes the terminal differentiation of embryonic muscle progenitors. Genes \& Development. 2008;22(5): 668-81.

32. Wang X, Niu Y, Zhou J, Zhu H, Ma B, Yu H et al. Crispr/cas9-mediated mstn disruption and heritable mutagenesis in goats causes increased body mass. Animal Genetics. 2018;49(1): 43-51.

33. Oldham JM, Martyn JAK, Sharma M, Jeanplong F, Kambadur R, Bass JJ. Molecular expression of myostatin and myod is greater in double-muscled than normal-muscled cattle fetuses. American Journal of Physiology-Regulatory, Integrative and Comparative Physiology. 2001;280(5): R1488-93.

34. Valdés JA, Flores S, Fuentes EN, Osorio-Fuentealba C, Jaimovich E, Molina A. Igf-1 induces ip3-dependent calcium signal involved in the regulation of myostatin gene expression mediated by nfat during myoblast 
differentiation. Journal of Cellular Physiology. 2013;228(7): 1452-63.

35. Yang W, Zhang Y, Li Y, Wu Z, Zhu D. Myostatin induces cyclin d1 degradation to cause cell cycle arrest through a phosphatidylinositol 3-kinase/akt/gsk-3beta pathway and is antagonized by insulin-like growth factor 1. Journal of Biological Chemistry. 2006;282(6): 3799-808.

36. Kurokawa M, Sato F, Aramaki S, Soh T, Yamauchi N, Hattori M. Monitor of the myostatin autocrine action during differentiation of embryonic chicken myoblasts into myotubes: effect of igf-i. Molecular and Cellular Biochemistry. 2009;331(1-2): 193-9.

37. McPherron ACLA. Regulation of skeletal muscle mass in mice by a new tgf-beta superfamily member. Nature. 1997; 387, 83-90(

38. Guo R, Wan Y, Xu D, Cui L, Deng M, Zhang G et al. Generation and evaluation of myostatin knock-out rabbits and goats using crispr/cas9 system. Scientific Reports. 2016;6(1): 29855.

39. Mc Pherron A C LSJ. Double muscling in cattle due to mutations in the myostatin gene. Proc Natl Acad Sci USA. 1997; 94: 12457-12461).

40. Wegner J, Albrecht E, Fiedler I, Teuscher F, Papstein HJ, Ender K. Growth- and breed-related changes of muscle fiber characteristics in cattle. Journal of Animal Science. 2000;78(6): 1485.

41. Swatland HJ. Muscle growth in the fetal and neonatal pig. Journal of Animal Science. 1973;37(2): 536-45.

42. Nishi M, Yasue A, Nishimatu S, Nohno T, Yamaoka T, Itakura M et al. A missense mutant myostatin causes hyperplasia without hypertrophy in the mouse muscle. Biochemical and Biophysical Research Communications. 2002;293(1): 247-51.

43. Zhu X, Hadhazy M, Wehling M, Tidball JG, McNally EM. Dominant negative myostatin produces hypertrophy without hyperplasia in muscle. FEBS Lett. 2000;474(1): 71-5.

44. He Z, Zhang T, Jiang L, Zhou M, Wu D, Mei J et al. Use of crispr/cas9 technology efficiently targetted goat myostatin through zygotes microinjection resulting in double-muscled phenotype in goats. Bioscience Reports. 2018;38(6).

45. Lv Q, Yuan L, Deng J, Chen M, Wang Y, Zeng J et al. Efficient generation of myostatin gene mutated rabbit by crispr/cas9. Scientific Reports. 2016;6(1): 25029.

46. Bi Y, Hua Z, Liu X, Hua W, Ren H, Xiao H et al. Isozygous and selectable marker-free mstn knockout cloned pigs generated by the combined use of crispr/cas9 and cre/loxp. Scientific Reports. 2016;6(1): 31729.

47. He Z, Zhang T, Jiang L, Zhou M, Wu D, Mei J et al. Use of crispr/cas9 technology efficiently targetted goat myostatin through zygotes microinjection resulting in double-muscled phenotype in goats. Biosci Rep. 2018;38(6).

\section{Figures}




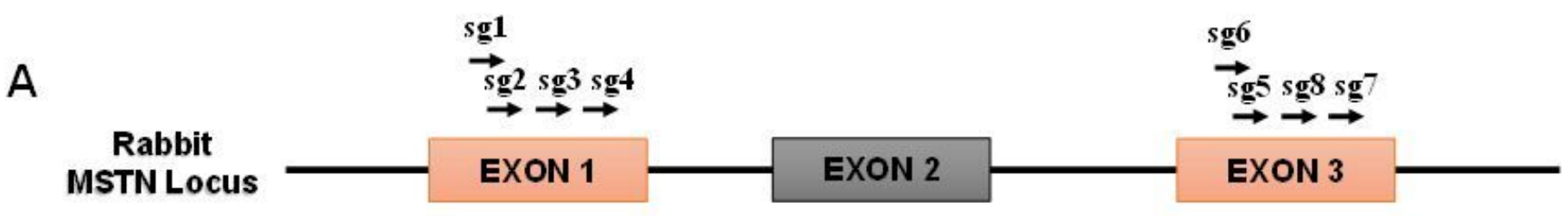

B
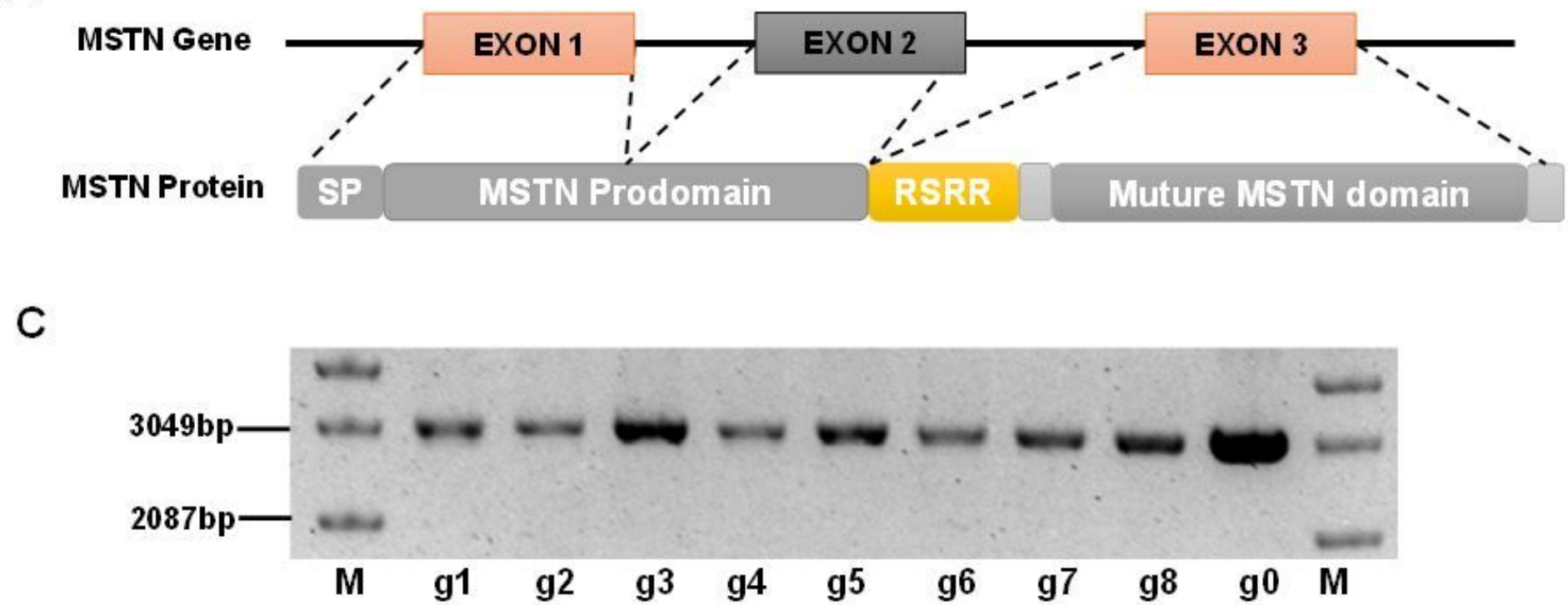

Figure 1

(A) Design and construction of sgRNA Schematic diagram of sgRNA targeting site in the rabbit MSTN gene locus. (B) Structure of GDF8 gene and MSTN protein. (C) Electropherogram of eight sgRNA vectors, MD Supercoiled DNA Ladder Marker,g1-g8 refer to phU6-gRNA-Sg1to phU6-gRNA-Sg8. 


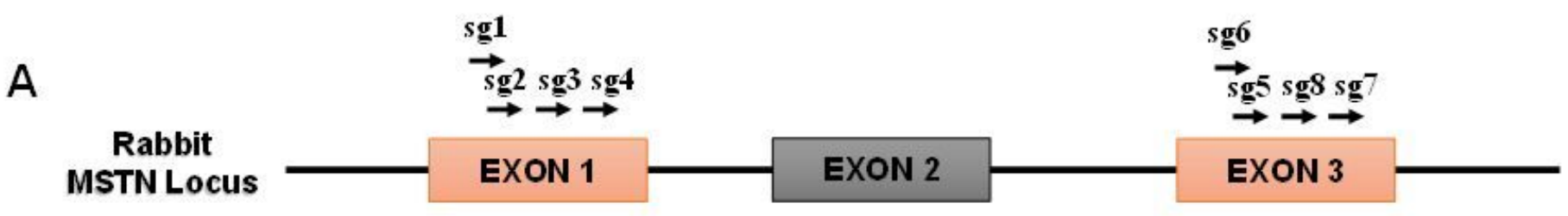

B
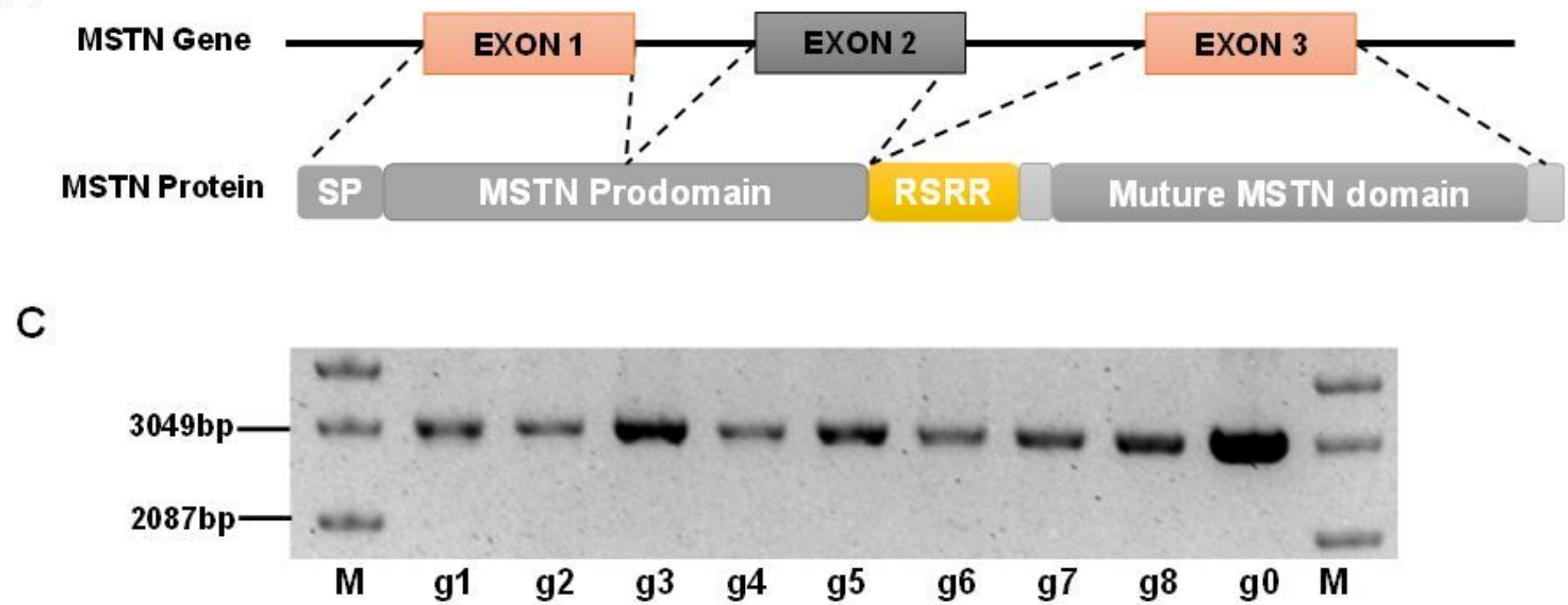

Figure 1

(A) Design and construction of sgRNA Schematic diagram of sgRNA targeting site in the rabbit MSTN gene locus. (B) Structure of GDF8 gene and MSTN protein. (C) Electropherogram of eight sgRNA vectors, MD Supercoiled DNA Ladder Marker,g1-g8 refer to phU6-gRNA-Sg1to phU6-gRNA-Sg8. 


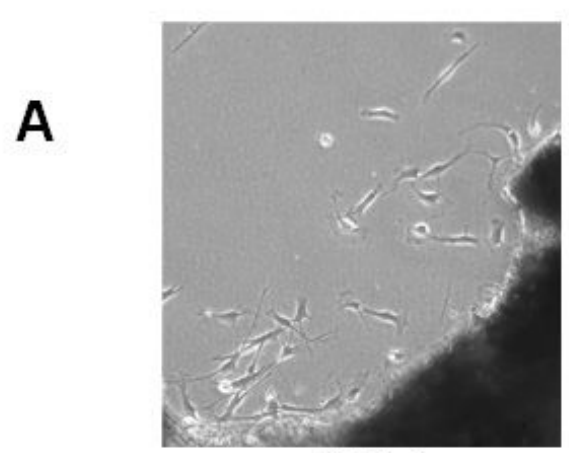

2DAY

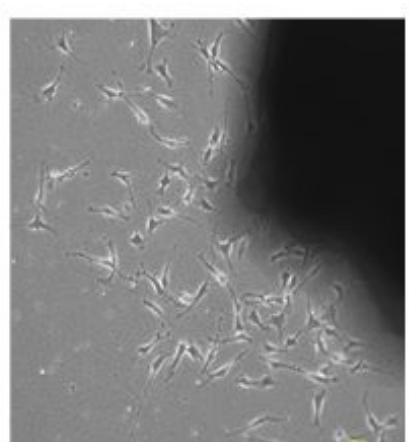

3DAY

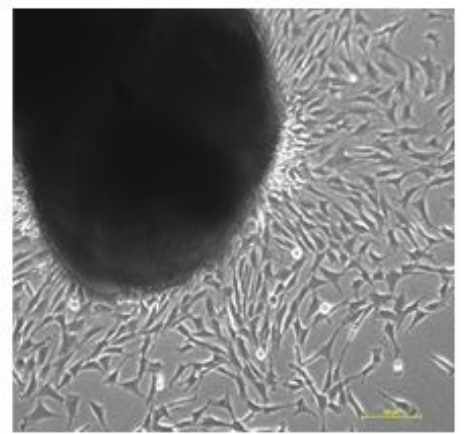

4DAY

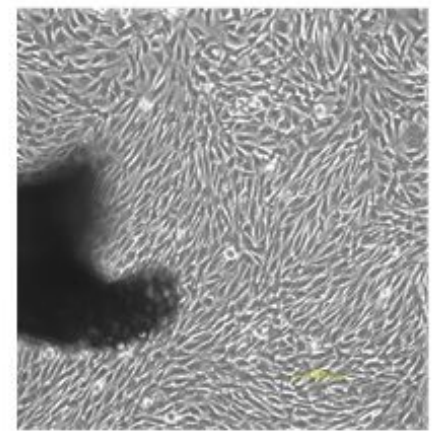

5DAY

B

WT IAAGACGGGCTGTGTAATGCATGCACTTGGAGACAAAACAGTAAATAT 0 g1 $(\mathbf{0}, \mathbf{0} \%)$ |AAGACGGGCTGTGTAATGCATGCACTTGGAGACAAAACAGTAAATAT 0 g2 $(\mathbf{0}, \mathbf{0} \%)$ |AAGACGGGCTGTGTAATGCATGCACTTGGAGACAAAACAGTAAATAT 0 g3 $(\mathbf{0}, \mathbf{0} \%)$ |AAGACGGGCTGTGTAATGCATGCACTTGGAGACAAAACAGTAAATAT 0 g4 $(0,0 \%)$ | AAGACGGGCTGTGTAATGCATGCACTTGGAGACAAAACAGTAAATAT 0

g5

$(\mathbf{5} / \mathbf{2 0}, \mathbf{2 5} \%)$ AAGAGATACAAGGCCAATTACT GCTCTGGAGAGTGTGAATTTGTG 0 AAGAGATACAAGGCCAATTACT--CTCTGGAGAGTGTGAATTTGTG $\quad-1$ AAGAGATACAAGGCCAATTACTGTTCTGGAGAGTGTGAATTTGTG C-T

g6 $(\mathbf{9} / \mathbf{2 0}, \mathbf{4 5} \%)$ TTGGATTATTGCGCCCAAGAGATACAAGGCCAATTACTGCTCTGG 0 TTGGATTATTGCGCCCAAG-----TACAAGGCCAATTACTGCTCTGG $\quad-3$ TTGGATTATTGCGCCCAAGAGATTACAAGGCCAATTACTGCTCTGG +1 TTGGATTATTGCGCCTAAGAGATACAAGGCCAATTACTGCTCTGG C-T TTGGATTATTGCGCCCAAGAG-TACAAGGCCAATTACTGCTCTGG $\quad-1$ TTGGATTATTGCGCCCAAGA-----CAAGGCCAATTACTGCTCTGG $\quad-4$

g7 $(\mathbf{6} / \mathbf{2 0}, \mathbf{3 0} \%)$ AATAATATATGGGAAAATTCCAGCCATGGTAGTAGACCGCTGTGG 0 AATAATATATGGGAAAATTCCA--CCATGGTAGTAGACCGCTGTGG $\quad-1$ AATAATATATGGGAAAATTCCA--.-- - TGGTAGTAGACCGCTGTGG $\quad$-4 AATAATATGTGGGAAAATTCCAGCCATGGTAGTAGACCGCTGTGG A-G AATAATATATGGGAAAATTCCAGCCCATGGTAGTAGACCGCTGTGG +1 AATAATATACGGGAAAATTCCAGCCATGGTAGTAGACCGCTGTGG T-C

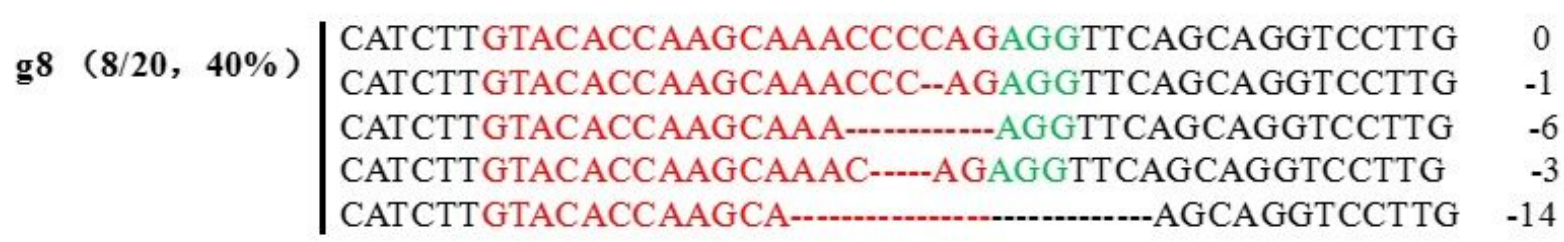

\section{Figure 2}

The results of sequencing for mutations at the specific sites in rabbit REFs. (A) Rabbit Primary fibroblast cell in culture from 2days to 5days. (B) Sanger sequence analysis of REFs. g1-g4 target to exon1, g5-g8 target to exon 3. gRNA edited were named on the left. g1-g8 represent different gRNA, PAM sequence is marked in green. The sgRNAs sequence is marked in red. The numbers on the right indicated the type of mutation, with "-" represents deletion of the given number of nucleotides, "+" represents insertion of the given number of nucleotides. 


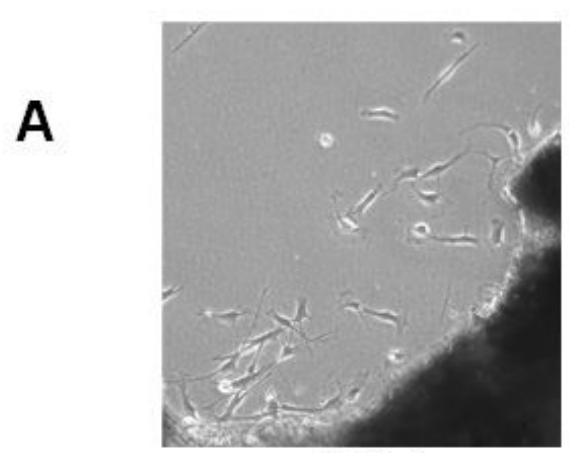

2DAY

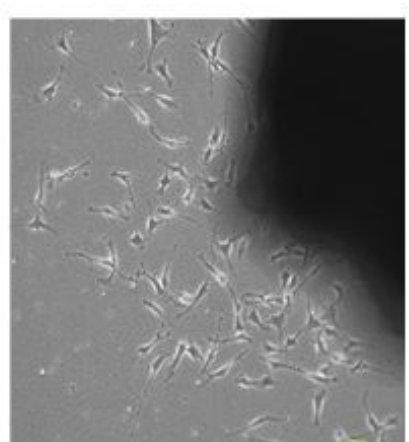

3DAY

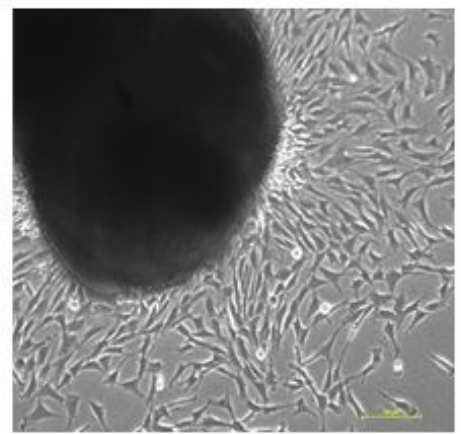

4DAY

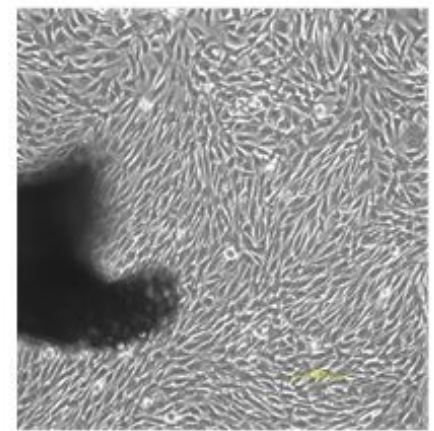

5DAY

B

WT IAAGACGGGCTGTGTAATGCATGCACTTGGAGACAAAACAGTAAATAT 0 g1 $(\mathbf{0}, \mathbf{0} \%)$ |AAGACGGGCTGTGTAATGCATGCACTTGGAGACAAAACAGTAAATAT 0 g2 $(\mathbf{0}, \mathbf{0} \%)$ |AAGACGGGCTGTGTAATGCATGCACTTGGAGACAAAACAGTAAATAT 0 g3 $(\mathbf{0}, \mathbf{0} \%)$ |AAGACGGGCTGTGTAATGCATGCACTTGGAGACAAAACAGTAAATAT 0 g4 $(0,0 \%)$ | AAGACGGGCTGTGTAATGCATGCACTTGGAGACAAAACAGTAAATAT 0

g5

$(\mathbf{5} / \mathbf{2 0}, \mathbf{2 5} \%)$ AAGAGATACAAGGCCAATTACT GCTCTGGAGAGTGTGAATTTGTG 0 AAGAGATACAAGGCCAATTACT--CTCTGGAGAGTGTGAATTTGTG $\quad-1$ AAGAGATACAAGGCCAATTACTGTTCTGGAGAGTGTGAATTTGTG C-T

g6 $(\mathbf{9} / \mathbf{2 0}, \mathbf{4 5} \%)$ TTGGATTATTGCGCCCAAGAGATACAAGGCCAATTACTGCTCTGG 0 TTGGATTATTGCGCCCAAG-----TACAAGGCCAATTACTGCTCTGG $\quad-3$ TTGGATTATTGCGCCCAAGAGATTACAAGGCCAATTACTGCTCTGG +1 TTGGATTATTGCGCCTAAGAGATACAAGGCCAATTACTGCTCTGG C-T TTGGATTATTGCGCCCAAGAG-TACAAGGCCAATTACTGCTCTGG $\quad-1$ TTGGATTATTGCGCCCAAGA-----CAAGGCCAATTACTGCTCTGG $\quad-4$

g7 $(\mathbf{6} / \mathbf{2 0}, \mathbf{3 0} \%)$ AATAATATATGGGAAAATTCCAGCCATGGTAGTAGACCGCTGTGG 0 AATAATATATGGGAAAATTCCA--CCATGGTAGTAGACCGCTGTGG $\quad-1$ AATAATATATGGGAAAATTCCA--.-- - TGGTAGTAGACCGCTGTGG $\quad$-4 AATAATATGTGGGAAAATTCCAGCCATGGTAGTAGACCGCTGTGG A-G AATAATATATGGGAAAATTCCAGCCCATGGTAGTAGACCGCTGTGG +1 AATAATATACGGGAAAATTCCAGCCATGGTAGTAGACCGCTGTGG T-C

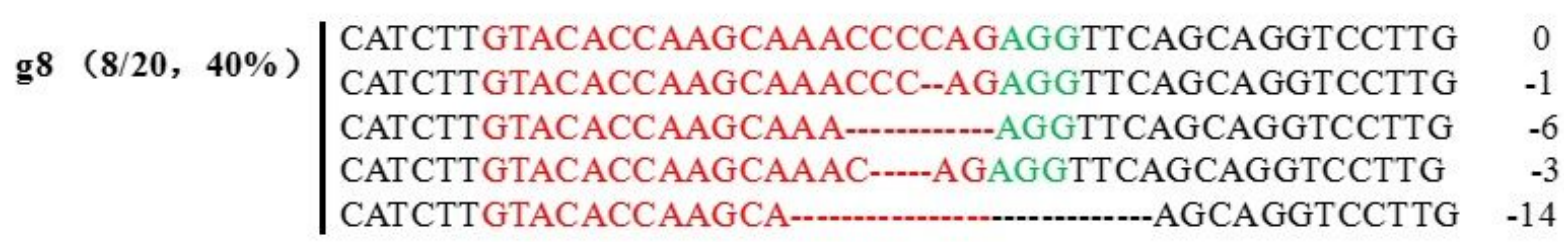

\section{Figure 2}

The results of sequencing for mutations at the specific sites in rabbit REFs. (A) Rabbit Primary fibroblast cell in culture from 2days to 5days. (B) Sanger sequence analysis of REFs. g1-g4 target to exon1, g5-g8 target to exon 3. gRNA edited were named on the left. g1-g8 represent different gRNA, PAM sequence is marked in green. The sgRNAs sequence is marked in red. The numbers on the right indicated the type of mutation, with "-" represents deletion of the given number of nucleotides, "+" represents insertion of the given number of nucleotides. 


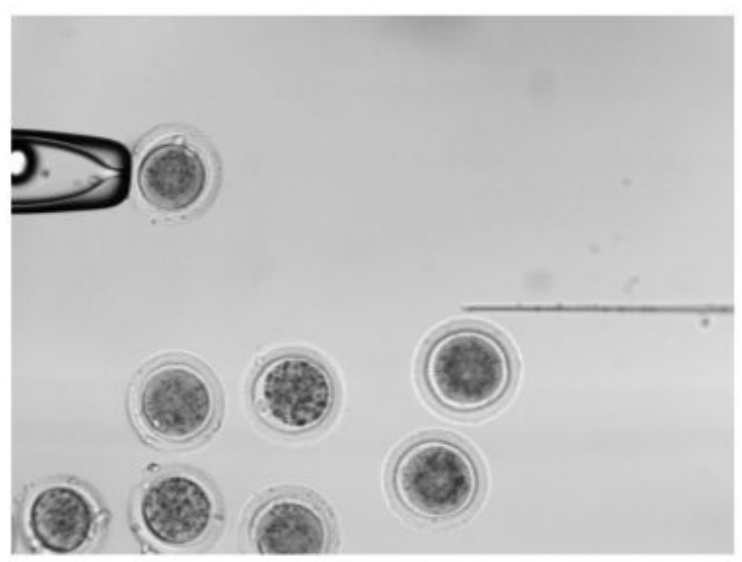

sgRNA 6

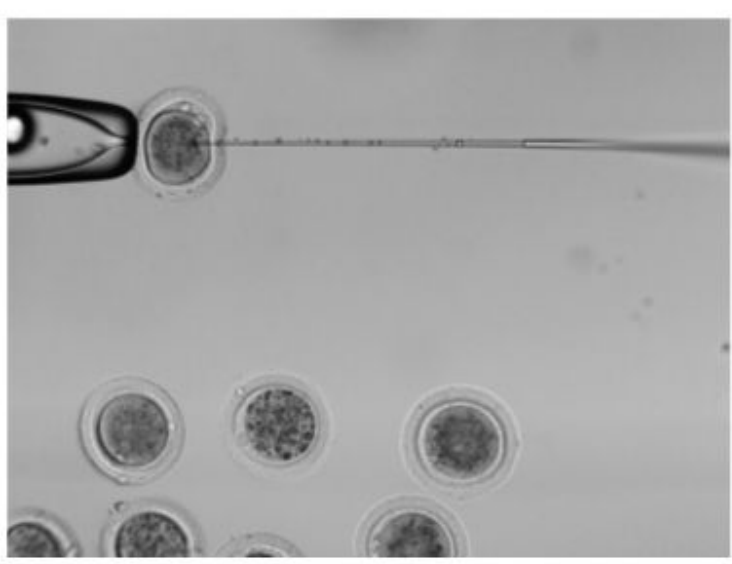

sgRNA8

wT I GGGATTGGATTATTGCGCCCAAGAGATACAAGGCCAATTACT// TTGTACACCAAGCAAACCCCAGAGGTTCAGC 0 BL1,BL6,BL7] GGGATTGGATTATTGCGCCCAAGAGATACAAGGCCAATTACT // TTGTACACCAAGCAAACCCCAGAGGTTCAGC 0

BL2 | GGGATTGGATTATTGCGCCCAAGAGATACAAGGCCAATTACT / TTGTACACCAAGCAAACCCCAGAGGTTCAGC 0 GGGATTGGATTATTGCGCCCAAGAGA-ACAAGGCCAATTACT // TTGTACACCAAGCAAACCCCAGAGGTTCAGC -1 GGGATTGGATTATTGCGCCCAAGAG-_-GCCAATTACT//TTGTACACCAAGCAAACCCCAGAGGTTCAGC -7 GGG--CCAATTACT//TTGTACACCAAGCAAACCCCAGAGGTTCAGC -30

BL3

GGGATTGGATTATTGCGCCCAAGAGATACAAGGCCAATTACT // TTGTACACCAAGCAAACCCCAGAGGTTCAGC 0 GGGATTGGATTATTGCGCCCAAG-_-_._GCCAATTACT//TTGTACACCAAGCAAACCCCAGAGGTTCAGC -9 GGGATTGGATTATTGCGCCCAA--_-TAACT//TTGTACACCAAGCAAACCCCAGAGGTTCAGC -15 GGGATTGGATTATTGCGCCCAAGAGA--ACAAGGCCAATTACT//TTGTACACCAAGCAAACCCCAGAGGTTCAGC -1 GGGATTGGATTATTGCGCCCAATA--_ACT//TTGTACACCAAGCAAACCC-AGAGGTTCAGC -16

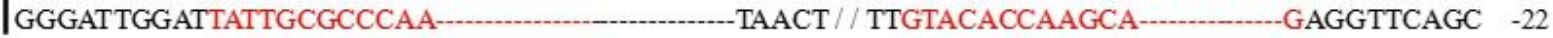

BL4,BL5 GGGATTGGATTATTGCGCCCAAG-_-_GCCAATTACT // TTGTACACCAAGCAAACCCCAGAGGTTCAGC -9 GGGATTGGATTATTGCGCCCAAG_-_-GCCAATTACT//TTGTACACCAAGCAAAC-CCAGAGGTTCAGC -10

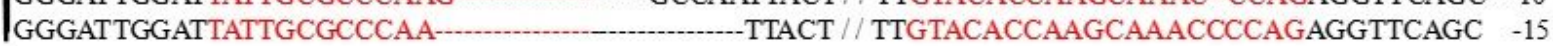

BLS

GGGATTGGATTATTGCGCCCAAGAGATACAAGGCCAATTACT // TTGTACACCAAGCAAACCCCAGAGGTTCAGC 0 GGGATTGGATTATTGCGCCCAAG-_-GCCAATTACT//TTGTACACCAAGCAAACCCCAGAGGTTCAGC -9 GGGATTGGATTATTGCGCCCAA-_._-TAACT//TTGTACACCAAGCAAACCCCAGAGGTTCAGC -15 GGGATTGGATTATTGCGCCCAAGAGA-ACAAGGCCAATTACT//TTGTACACCAAGCAAACCCCAGAGGTTCAGC -1 GGGATTGGATTATTGCGCCCAATA-_-_ACT // TTGTACACCAAGCAAACCC-AGAGGTTCAGC -16

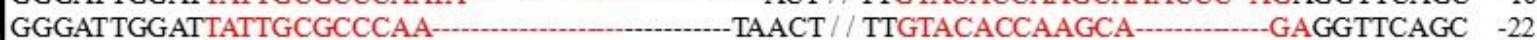

BL9

GGGATTGGATTATTGCGCCCAAG GCCAATTACT // TTGTACACCAAGCAAACCCCAGAGGTTCAGC -9 GGGATTGGATTATTGCGCCCAAGAGA--ACAAGGCCAATTACT//TTGTACACCAAGCAAACCCCAGAGGTTCAGC -1 GGGATTGGATTATTGCGCCCAAGAG_________CCAATTACT //TTGTACACCAAGCAAACCCCAGAGGTTCAGC -7

BL10 GGGATTGGATTATTGCGCCCAAGAGA-ACAAGGCCAATTACT// TTGTACACCAAGCAAACCCCAGAGGTTCAGCAGG -1

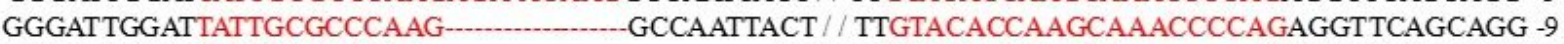
GGGATTGGATTATTGCGCCCAA-_-_-ACT//TTGTACACCAAGCA_-_-_GAGGTCAGCAGG -22

\section{Figure 3}

Targeted mutagenesis efficiency of the CRISPR/Cas9 system in rabbit zygotes. (A) Schematic diagram of the microinjection of Cas9 mRNA and sgRNAs into rabbit zygotes. (B) BL1-BL7 represent different blastocysts used in this study. Sanger sequence analysis of zygotes. zygotes edited were named on the left. PAM sequence is marked in green. The sgRNAs sequence is marked in red. The numbers on the right indicated the type of mutation, with "-" represents deletion of the given number of nucleotides. 


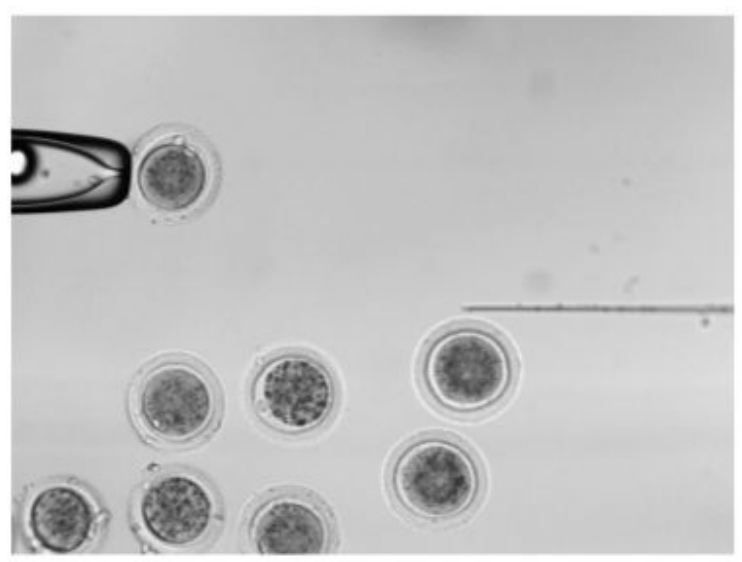

sgRNA 6

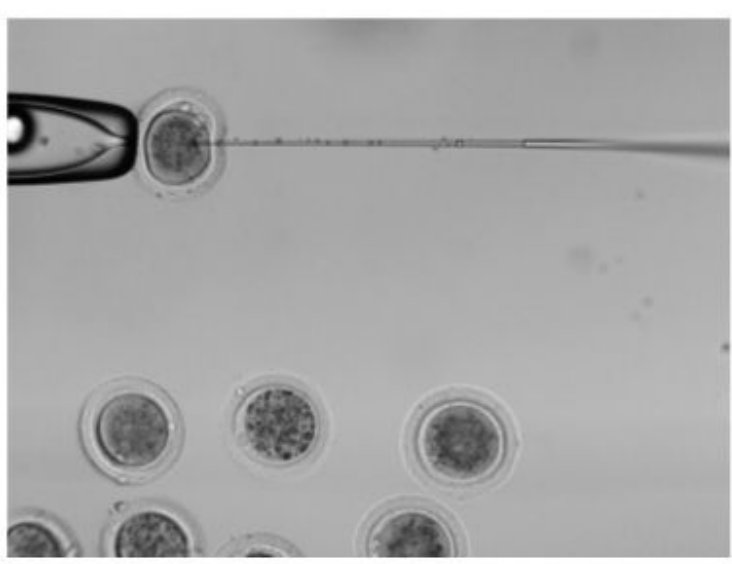

sgRNA8

wT I GGGATTGGATTATTGCGCCCAAGAGATACAAGGCCAATTACT// TTGTACACCAAGCAAACCCCAGAGGTTCAGC 0 BL1,BL6,BL7] GGGATTGGATTATTGCGCCCAAGAGATACAAGGCCAATTACT // TTGTACACCAAGCAAACCCCAGAGGTTCAGC 0

BL2 | GGGATTGGATTATTGCGCCCAAGAGATACAAGGCCAATTACT / TTGTACACCAAGCAAACCCCAGAGGTTCAGC 0 GGGATTGGATTATTGCGCCCAAGAGA-ACAAGGCCAATTACT // TTGTACACCAAGCAAACCCCAGAGGTTCAGC -1 GGGATTGGATTATTGCGCCCAAGAG-_-GCCAATTACT//TTGTACACCAAGCAAACCCCAGAGGTTCAGC -7 GGG--CCAATTACT//TTGTACACCAAGCAAACCCCAGAGGTTCAGC -30

BL3

GGGATTGGATTATTGCGCCCAAGAGATACAAGGCCAATTACT // TTGTACACCAAGCAAACCCCAGAGGTTCAGC 0 GGGATTGGATTATTGCGCCCAAG-_-_._GCCAATTACT//TTGTACACCAAGCAAACCCCAGAGGTTCAGC -9 GGGATTGGATTATTGCGCCCAA--_-TAACT//TTGTACACCAAGCAAACCCCAGAGGTTCAGC -15 GGGATTGGATTATTGCGCCCAAGAGA--ACAAGGCCAATTACT//TTGTACACCAAGCAAACCCCAGAGGTTCAGC -1 GGGATTGGATTATTGCGCCCAATA--_ACT//TTGTACACCAAGCAAACCC-AGAGGTTCAGC -16

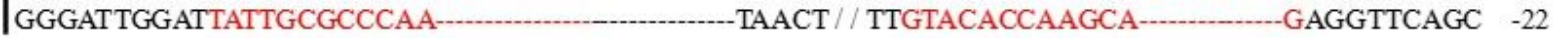

BL4,BL5 GGGATTGGATTATTGCGCCCAAG-_-_GCCAATTACT // TTGTACACCAAGCAAACCCCAGAGGTTCAGC -9 GGGATTGGATTATTGCGCCCAAG_-_-GCCAATTACT//TTGTACACCAAGCAAAC-CCAGAGGTTCAGC -10

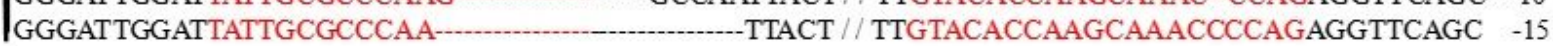

BLS

GGGATTGGATTATTGCGCCCAAGAGATACAAGGCCAATTACT // TTGTACACCAAGCAAACCCCAGAGGTTCAGC 0 GGGATTGGATTATTGCGCCCAAG-_-GCCAATTACT//TTGTACACCAAGCAAACCCCAGAGGTTCAGC -9 GGGATTGGATTATTGCGCCCAA-_._-TAACT//TTGTACACCAAGCAAACCCCAGAGGTTCAGC -15 GGGATTGGATTATTGCGCCCAAGAGA-ACAAGGCCAATTACT//TTGTACACCAAGCAAACCCCAGAGGTTCAGC -1 GGGATTGGATTATTGCGCCCAATA-_-_ACT // TTGTACACCAAGCAAACCC-AGAGGTTCAGC -16

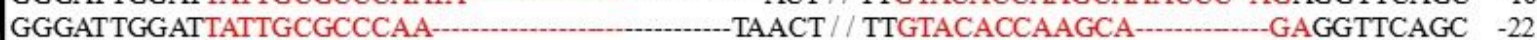

BL9

GGGATTGGATTATTGCGCCCAAG GCCAATTACT // TTGTACACCAAGCAAACCCCAGAGGTTCAGC -9 GGGATTGGATTATTGCGCCCAAGAGA--ACAAGGCCAATTACT//TTGTACACCAAGCAAACCCCAGAGGTTCAGC -1 GGGATTGGATTATTGCGCCCAAGAG_________CCAATTACT //TTGTACACCAAGCAAACCCCAGAGGTTCAGC -7

BL10 GGGATTGGATTATTGCGCCCAAGAGA-ACAAGGCCAATTACT// TTGTACACCAAGCAAACCCCAGAGGTTCAGCAGG -1

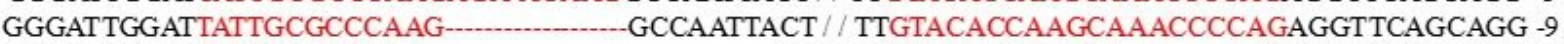
GGGATTGGATTATTGCGCCCAA-_-_-ACT//TTGTACACCAAGCA_-_-_GAGGTCAGCAGG -22

\section{Figure 3}

Targeted mutagenesis efficiency of the CRISPR/Cas9 system in rabbit zygotes. (A) Schematic diagram of the microinjection of Cas9 mRNA and sgRNAs into rabbit zygotes. (B) BL1-BL7 represent different blastocysts used in this study. Sanger sequence analysis of zygotes. zygotes edited were named on the left. PAM sequence is marked in green. The sgRNAs sequence is marked in red. The numbers on the right indicated the type of mutation, with "-" represents deletion of the given number of nucleotides. 


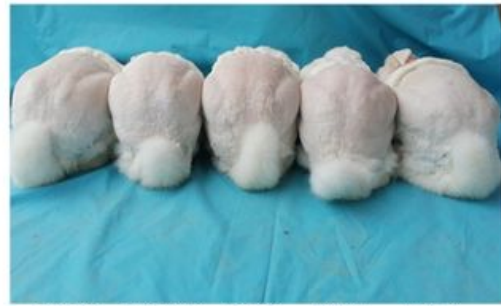

M2-1 M15-2 T2-2 $\quad$ T7-1 $\quad$ T7-4

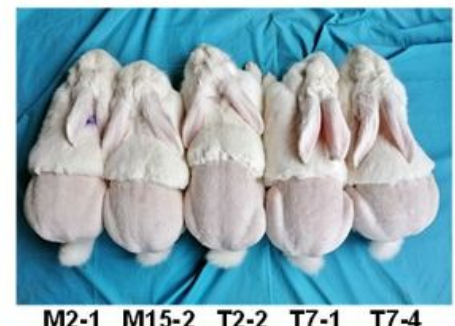

M2-1 M15-2 T2-2 T7-1 T7-4

B

sgRNA 6

SgRNA8

wT I GGGATTGGATTATTGCGCCCAAGAGATACAAGGCCAATTACT// TTGTACACCAAGCAAACCCCAGAGGTTCAGC

M2-1 GGGATTGGATTATTGCGCCCAAGAGATACAAGGCCAATTACT//TTGTACACCAAGCAAACCCCAGAGGTTCAGC 0 GGGATTGGATTATTGCGCCCAAG-............-GCCAATTACT//TTGTACACCAAGCAAACCCCAGAGGTTCAGC

GGGATTGGATTATTGCGCCCAAGAGA-ACAAGGCCAATTACT//TTGTACACCAAGCAAACCCCAGAGGTTCAGC -1

15-2 GGGATTGGATTATTGCGCCCAAGAGATACAAGGCCAATTACT / /TTGTACACCAAGCAAACCCCAGAGGTTCAGC GGGATTGGATTATTGCGCCCAAGAGA-ACAAGGCCAATTACT//TTGTACACCAAGCAAACCCCAGAGGTTCAGC GGGATTGGATTATTGCGCCCAAGAG-..............CCAATTACT//TTGTACACCAAGCAAACCCCAGAGGTTCAGC GGGATTGGATTATTGCGCCCAAG.................CCAATTACT //TTGTACACCAAGCAAACCCCAGAGGTCAGC

T2-2 GGGATTGGATTATTGCGCCCAAGAGA-ACAAGGCCAATTACT//TTGTACACCAAGCAAACCCCAGAGGTTCAGC GGGATTGGATTATTGCGCCCAAGAG.............-GCCAATTACT //TTGTACACCAAGCAAACCCCAGAGGTCAGC -

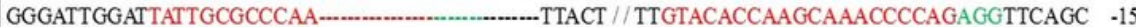
GGGATTGGATTATTGCGCCCAA

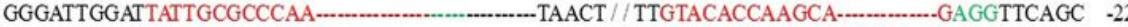

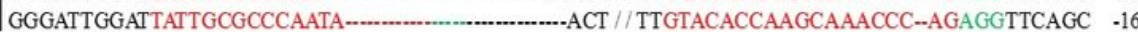

T7-1 GGGATTGGATTATTGCGCCCAAGAGATACAAGGCCAATTACT / TTGTACACCAAGCAAACCCCAGAGGTTCAGC GGGATTGGATTATTGCGCCCAAG_.................CCAATTACT//TTGTACACCAAGCAAACCCCAGAGGTTCAGC -9 GGGATTGGATTATT GCGCCCAA_..............................

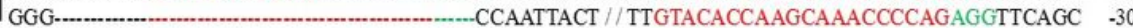

T7-4 GGGATTGGATTATTGCGCCCAAG-.......-GCCAATTACT// TTGTACACCAAGCAAACCCCAGAGGTCAGC -9 GGGATTGGATTATTGCGCCCAAGAGA-ACAAGGCCAATTACT//TTGTACACCAAGCAAACCCCAGAGGTTCAGC -1

C
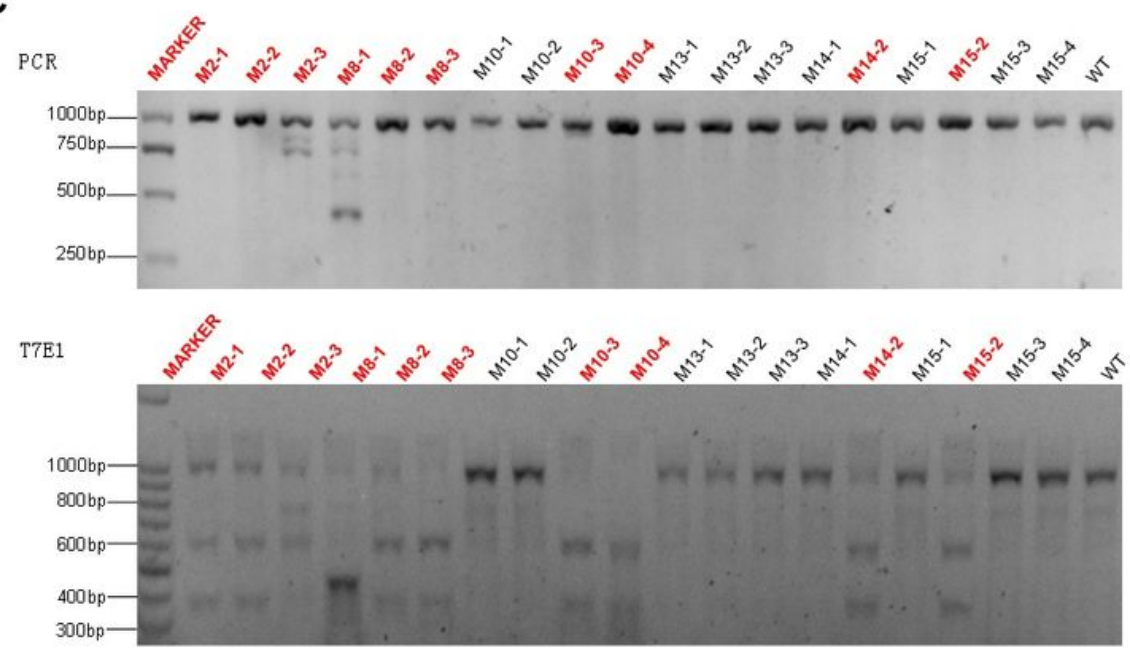

\section{Figure 4}

Generation of F0 MSTN KO rabbits via CRISPR/Cas9. (A) Five mutated rabbits of F0. (B) Sanger sequence analysis of mutated rabbits. Five rabbits edited were named on the left. WT stands for wild type control, PAM sequence is marked in green. The sgRNAs sequence is marked in red. The numbers on the right indicated the type of mutation, with "-" represents deletion of the given number of nucleotides. (C) Upper: The results of sequencing for mutations at the specific sites in Agarose gel electrophoresis of PCR products about a part of pups of F0. Lower: T7E1 cleavage assay for the rabbits. 


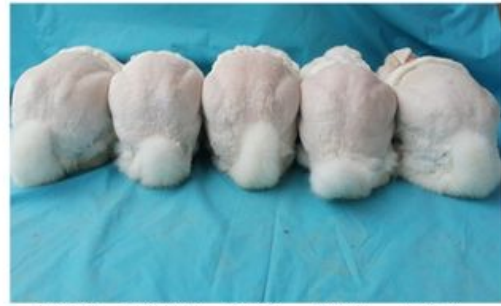

M2-1 M15-2 T2-2 $\quad$ T7-1 $\quad$ T7-4

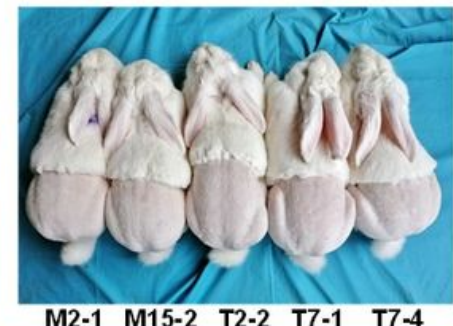

SgRNA8

M2-1 M15-2 T2-2 T7-1 T7-4

B

sgRNA 6

\begin{tabular}{|c|c|c|}
\hline & sgRNA 6 & \\
\hline WT & | GGGATTGGATTATTGCGCCCAAGAGATACAAGGCCAATTACT// TTGTACACCAAGCAAACCCCAGAGGTTCAGC & 0 \\
\hline M2-1 & 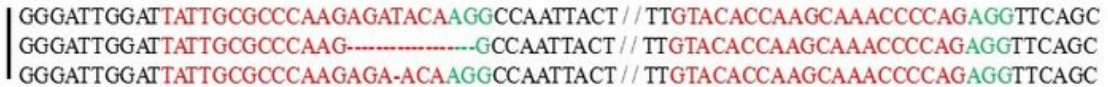 & $\begin{array}{l}0 \\
-9 \\
-1\end{array}$ \\
\hline M15-2 & 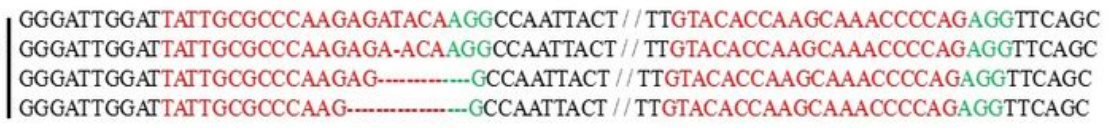 & $\begin{array}{l}0 \\
-1 \\
-7 \\
-9\end{array}$ \\
\hline T2-2 & $\begin{array}{l}\text { GGGATTGGATTATTGCGCCCAAGAGA-ACAAGGCCAATTACT / / TTGTACACCAAGCAAACCCCAGAGGTTCAGC } \\
\text { GGGATTGGATTATTGCGCCCAAGAG- } \\
\text { GGGATTGGATTATTGCGCCCAA- } \\
\text { GGGATTGGATTATTGCGCCCAA } \\
\text { GGGATTGGATTATTGCGCCCAA- } \\
\text { GGGATTGGATTATTGCGCCCAATA }\end{array}$ & $\begin{array}{l}-1 \\
-7 \\
-15 \\
-22 \\
-22 \\
-16\end{array}$ \\
\hline
\end{tabular}

T7-1 GGGATTGGATTATTGCGCCCAAGAGATACAAGGCCAATTACT//TTGTACACCAAGCAAACCCCAGAGGTCAGC GGGATTGGATTATTGCGCCCAAG_......................... GGGATTGGATTATTGCGCCCAA_....................... GGG_.........................

T7-4 GGGATTGGATTATTGCGCCCAAG-................CCAATTACT// TTGTACACCAAGCAAACCCCAGAGGTCAGC -9 GGGATTGGATTATTGCGCCCAAGAGA-ACAAGGCCAATTACT//TTGTACACCAAGCAAACCCCAGAGGTTCAGC -1

C
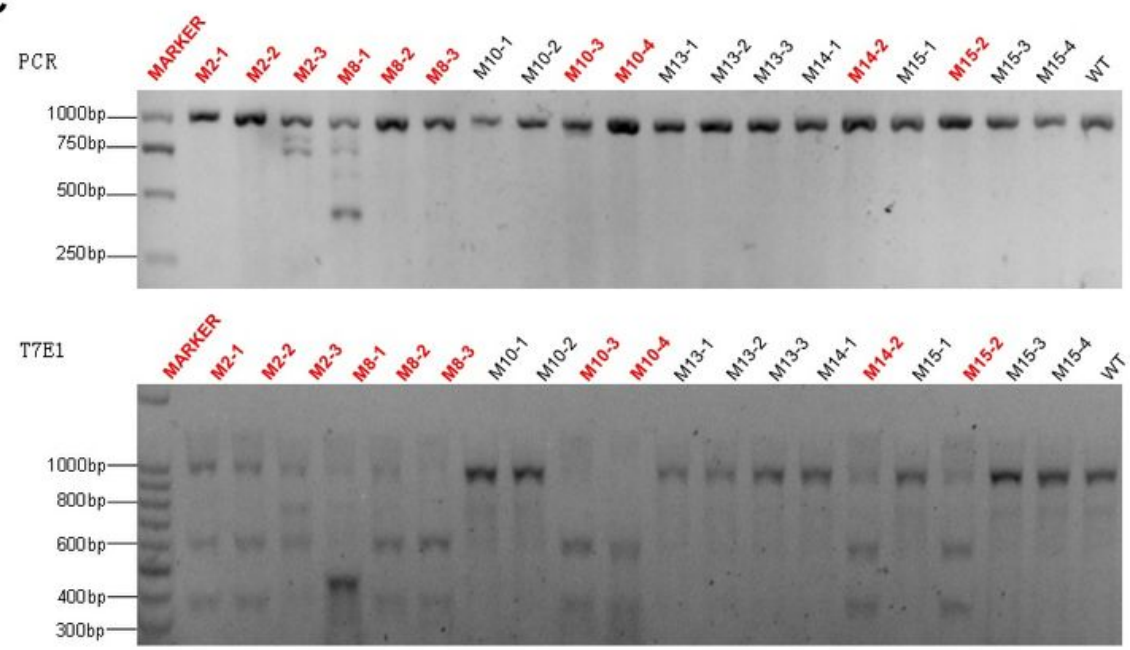

\section{Figure 4}

Generation of FO MSTN KO rabbits via CRISPR/Cas9. (A) Five mutated rabbits of F0. (B) Sanger sequence analysis of mutated rabbits. Five rabbits edited were named on the left. WT stands for wild type control, PAM sequence is marked in green. The sgRNAs sequence is marked in red. The numbers on the right indicated the type of mutation, with "-" represents deletion of the given number of nucleotides. (C) Upper: The results of sequencing for mutations at the specific sites in Agarose gel electrophoresis of PCR products about a part of pups of F0. Lower: T7E1 cleavage assay for the rabbits. 
A

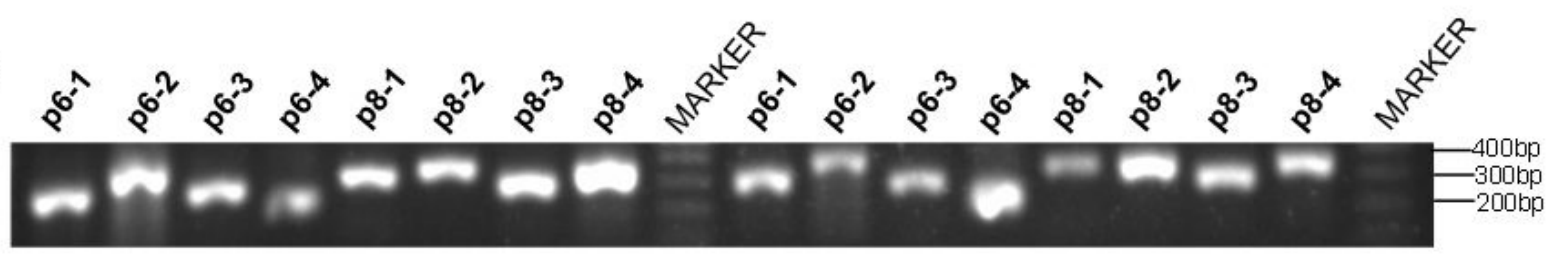

B

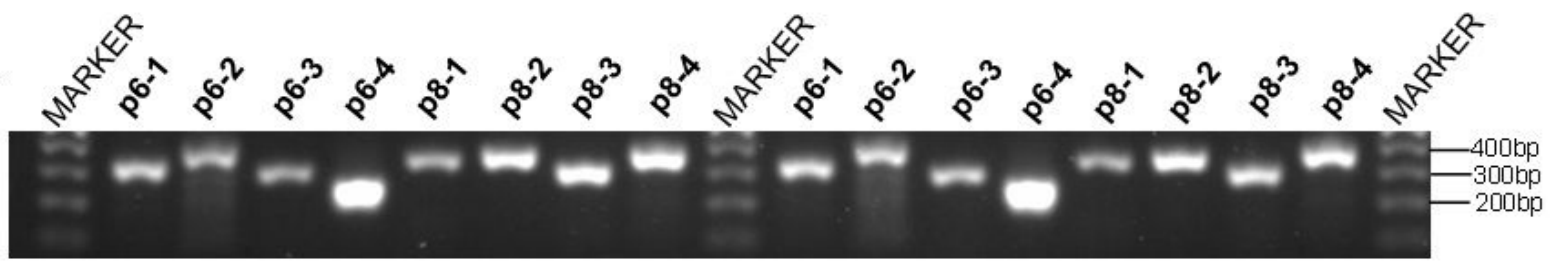

C

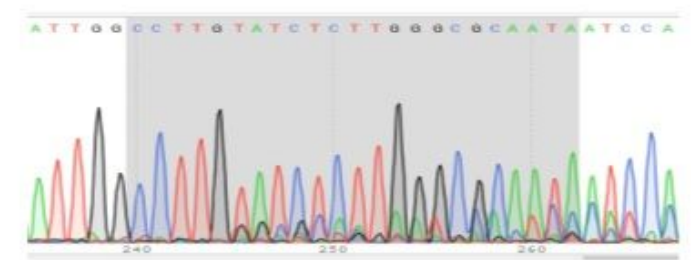

POTS 6-1

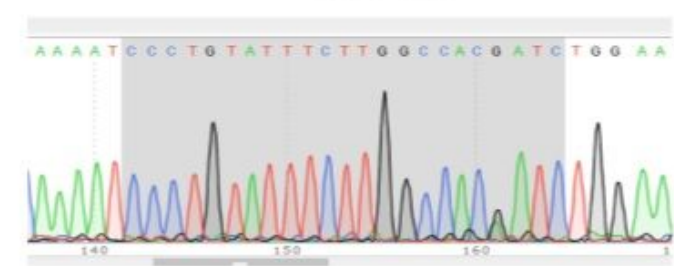

POTS 6-2

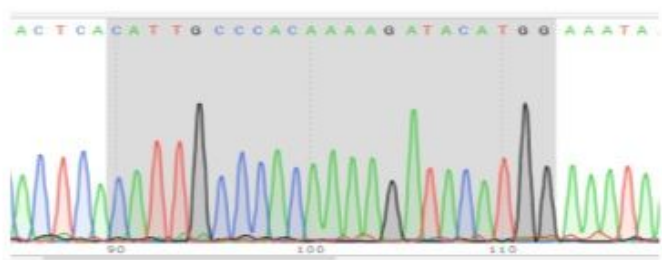

POTS 6-3

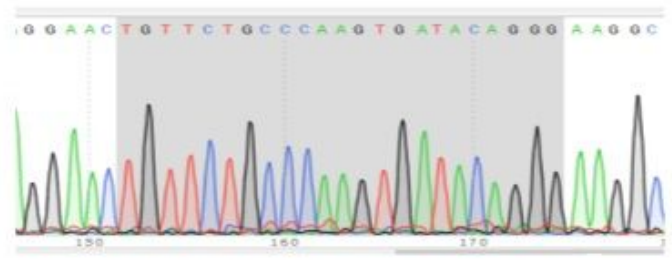

POTS 6-4

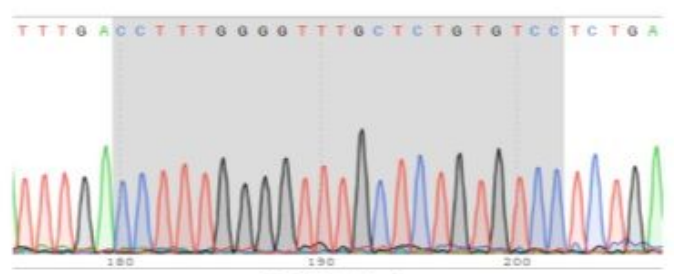

POTS 8-1

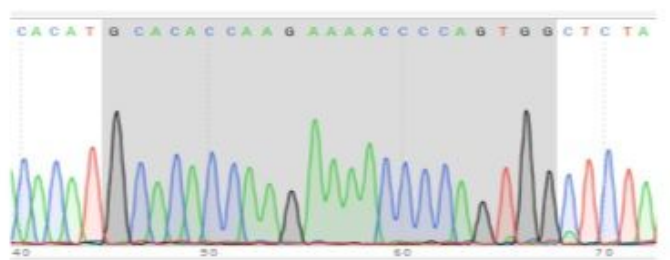

POTS $8-2$

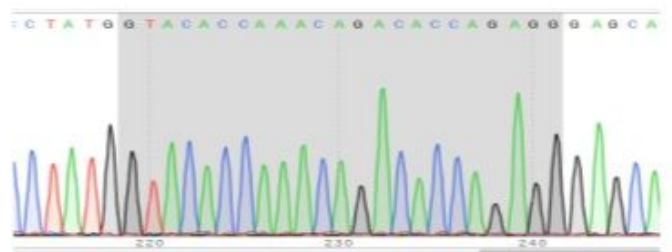

POTS $8-3$

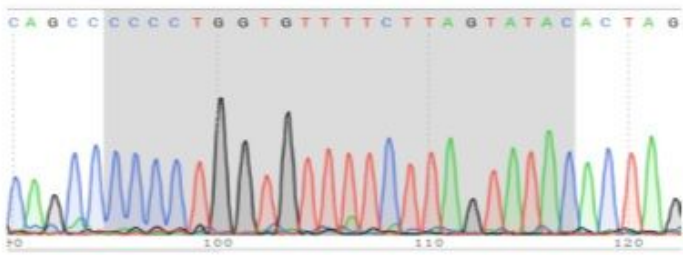

POTS $8-4$

\section{Figure 5}

Detection of MSTN modifiled rabbits. (A) The results of suspicious POTs in Agarose gel electrophoresis of PCR products. M: DL100bp; p6-1 p6-4 represent g6 four suspicious POTs, p8-1 p8-4 represent g8 four suspicious POTs. (B) The results of suspicious POTs in Agarose gel electrophoresis of T7E1. (C) Sanger sequencing for suspicious POTs sites. 
A

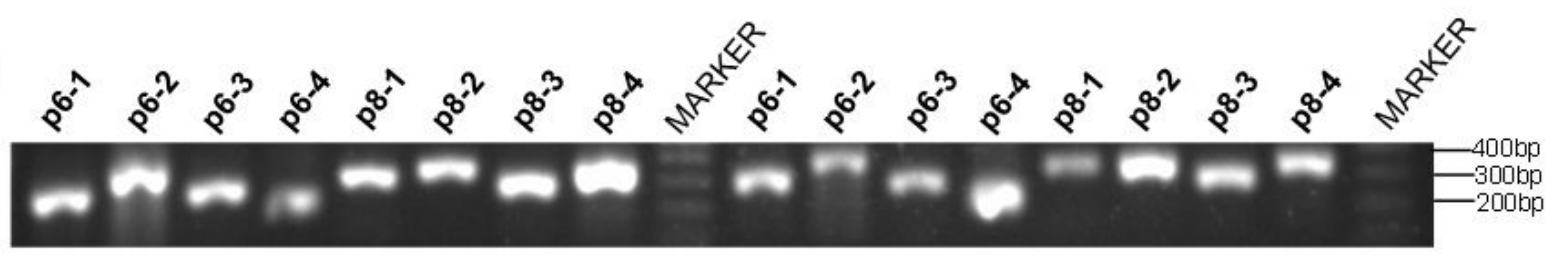

B

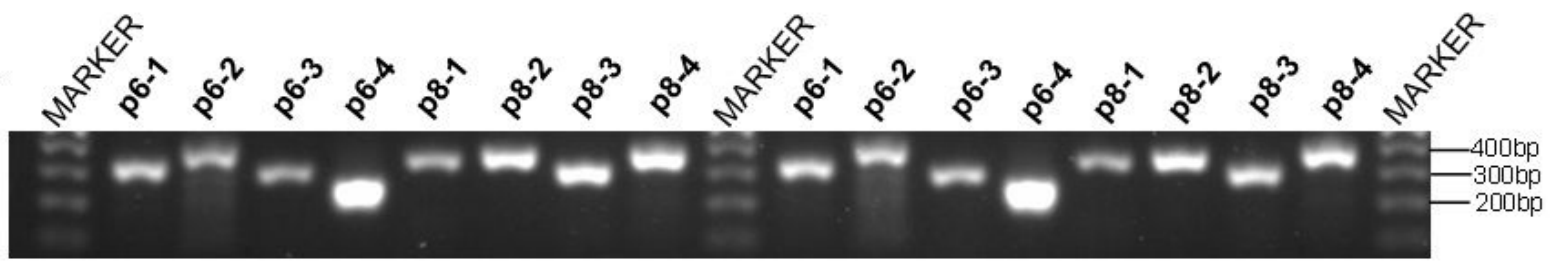

C

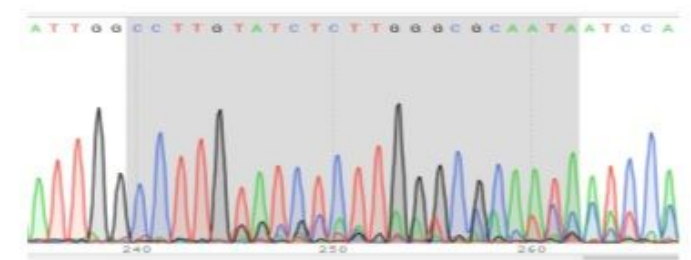

POTS 6-1

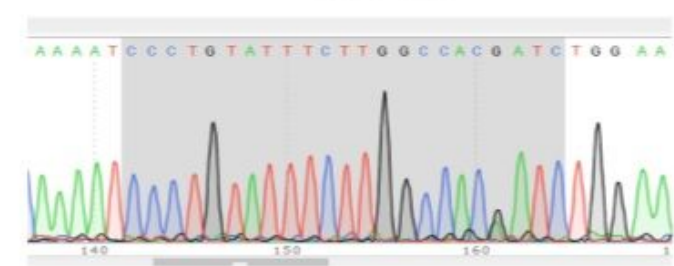

POTS 6-2

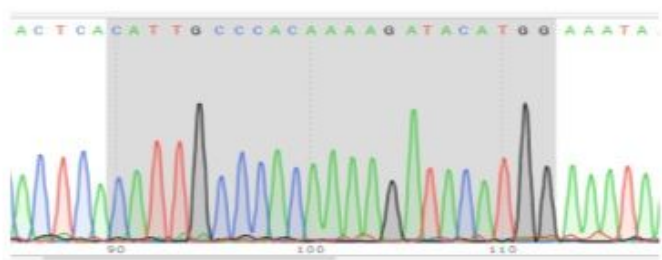

POTS 6-3

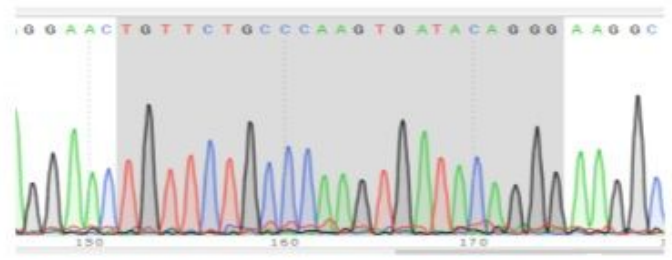

POTS 6-4

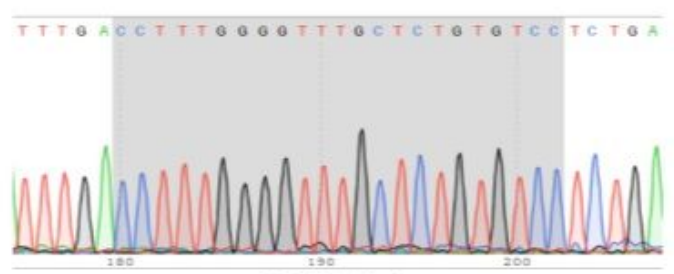

POTS 8-1

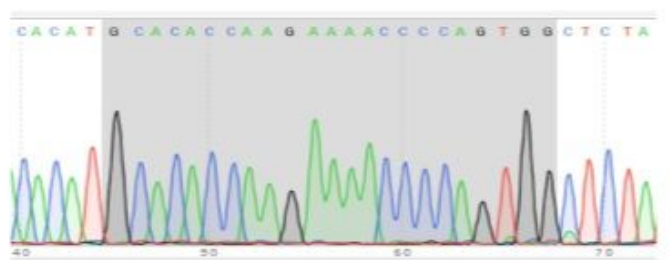

POTS $8-2$

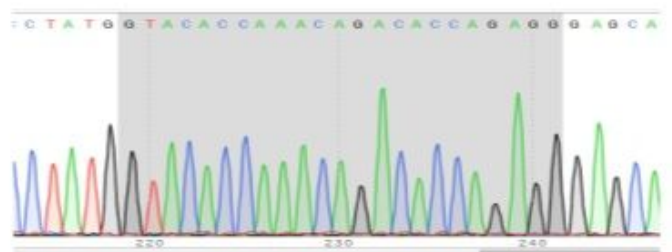

POTS $8-3$

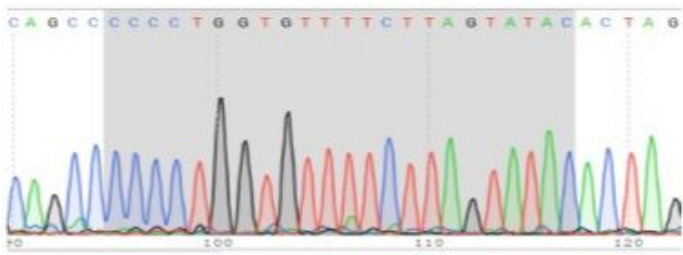

POTS $8-4$

Figure 5

Detection of MSTN modifiled rabbits. (A) The results of suspicious POTs in Agarose gel electrophoresis of PCR products. M: DL100bp; p6-1 p6-4 represent g6 four suspicious POTs, p8-1 p8-4 represent g8 four suspicious POTs. (B) The results of suspicious POTs in Agarose gel electrophoresis of T7E1. (C) Sanger sequencing for suspicious POTs sites. 


\section{WT $\quad$ MSTN $^{+-} \quad$ MSTN $^{-/-}$}

\section{Myostatin}

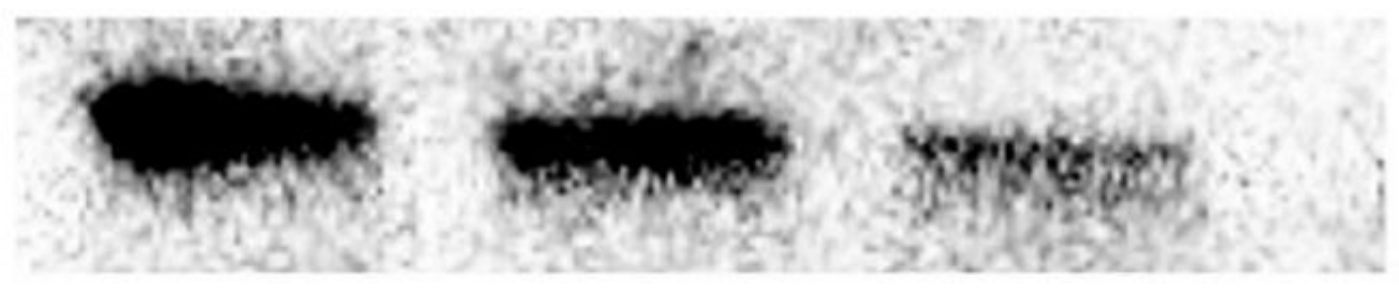

\section{$\beta$-actin}

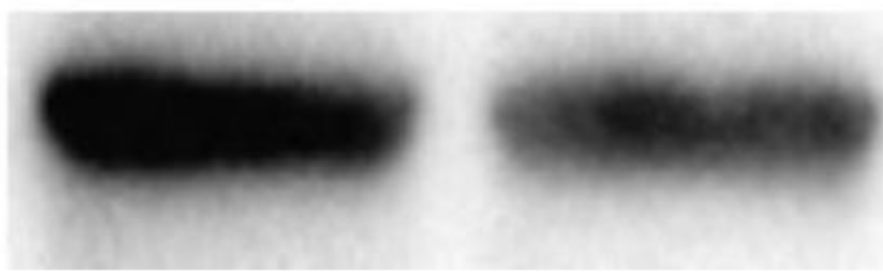

Figure 6

Result of MSTN+/-, MSTN-/- and WT rabbits in Western Blot. Beta-actin served as a loading

\section{Myostatin}

\section{WT \\ $\mathrm{MSTN}^{+/-}$ \\ MSTN $^{-/-}$}

\section{$\beta$-actin}
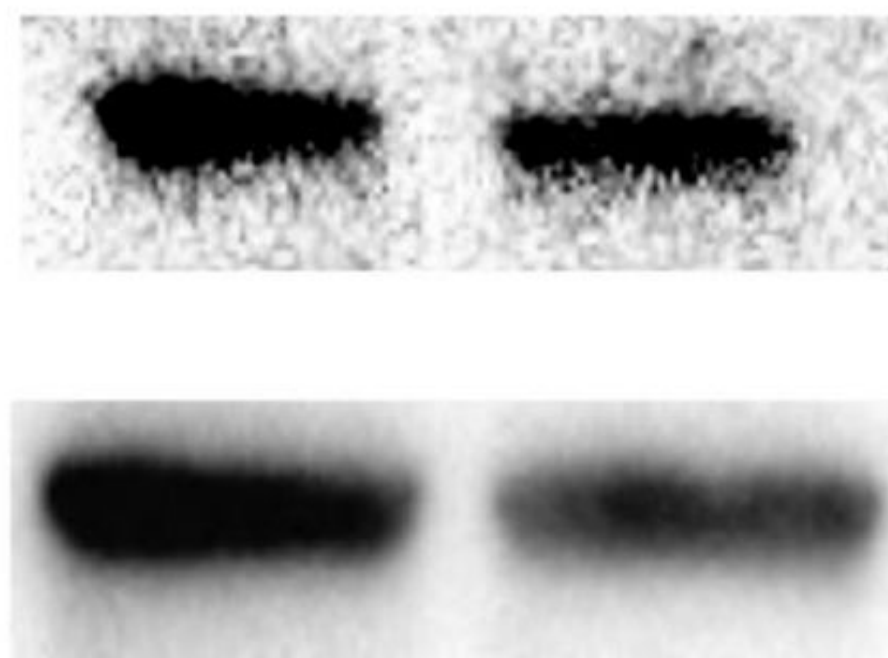

Figure 6

Result of MSTN+/-, MSTN-/- and WT rabbits in Western Blot. Beta-actin served as a loading 

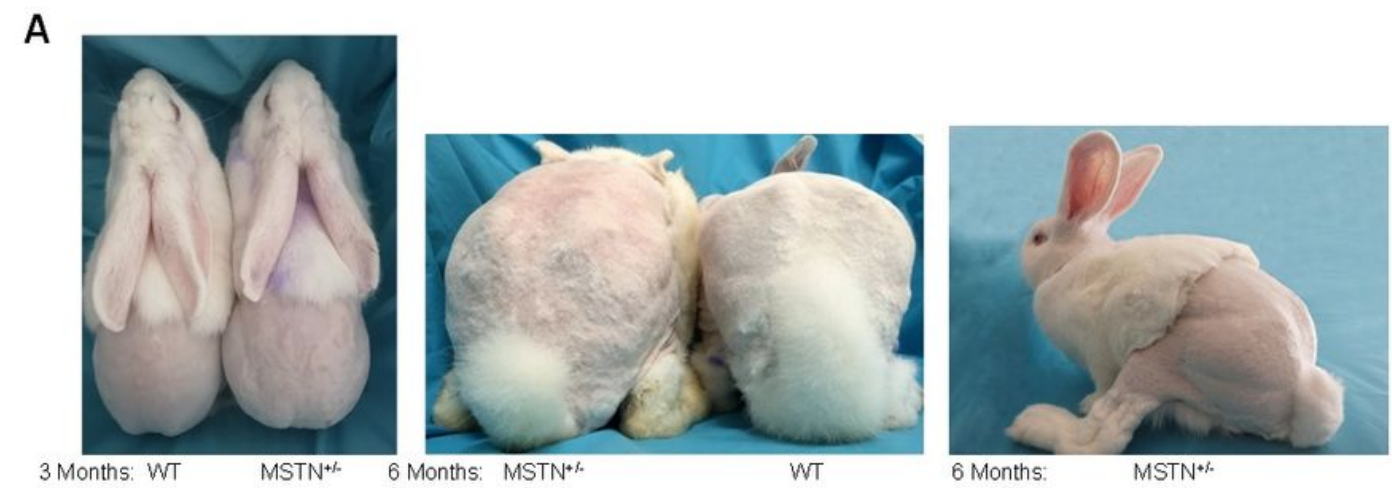

B
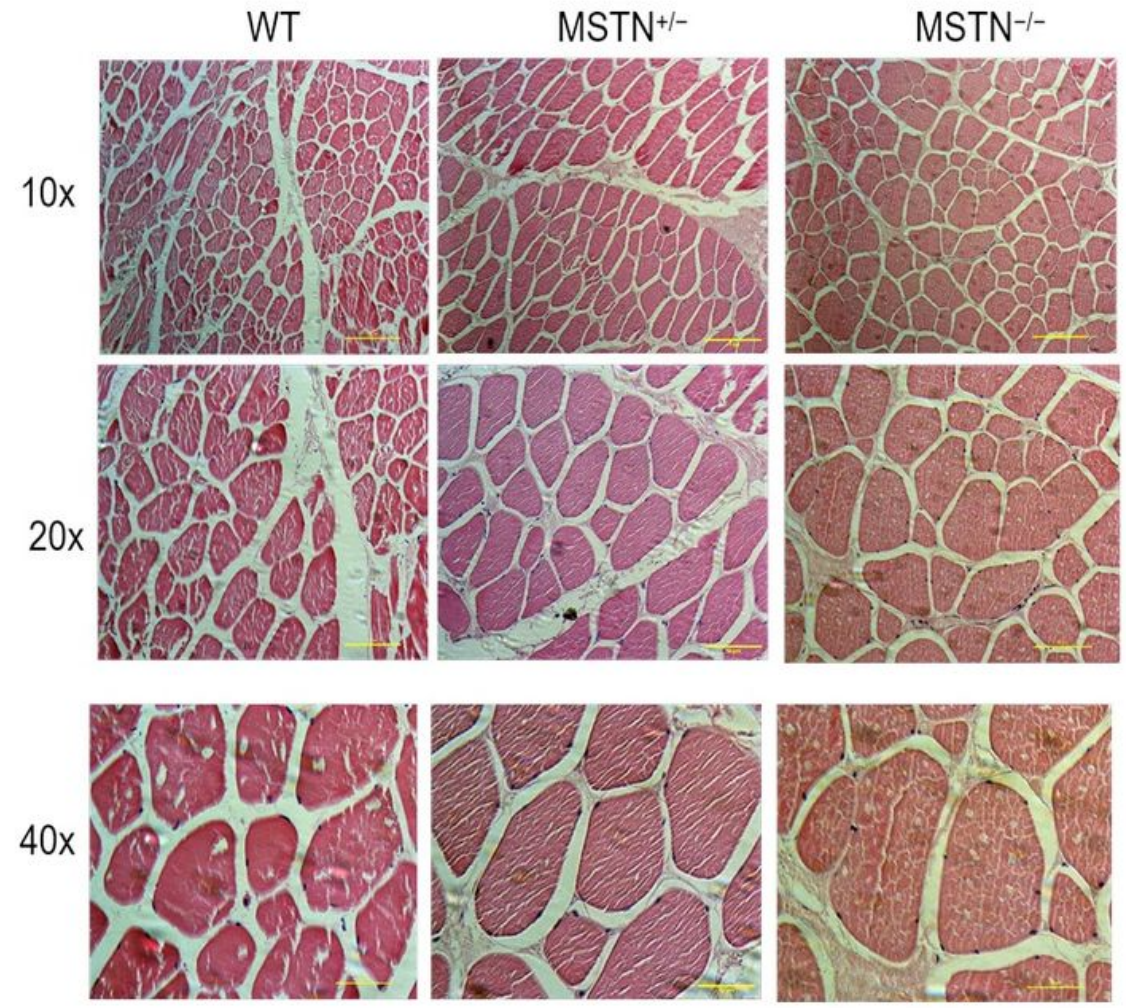

C

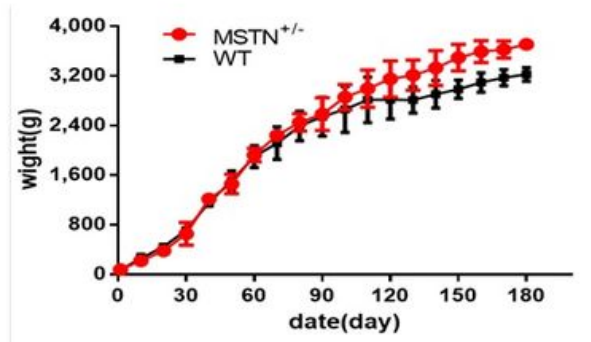

\section{Figure 7}

Detection and observation of MSTN-KO rabbits. (A) Comparison of mediated and wild type Rabbits at 3 and 6 months of age. (B) H\&E staining of the muscle fibers from gluteus maximus. (C) The average body weight of MSTN+/- and WT rabbits from FO $(n=5)$. 

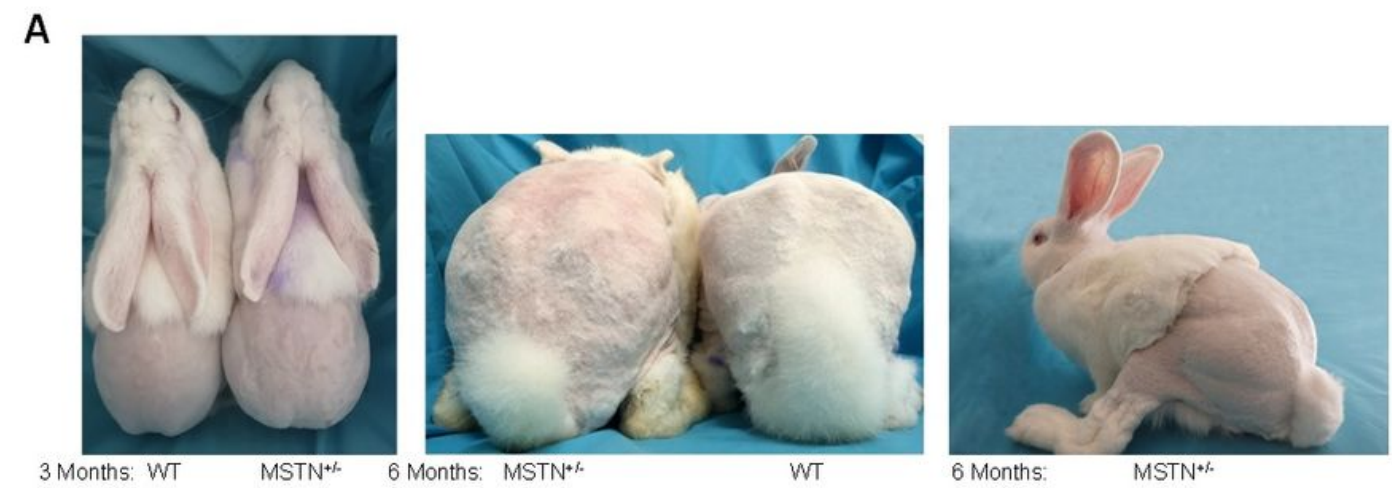

B
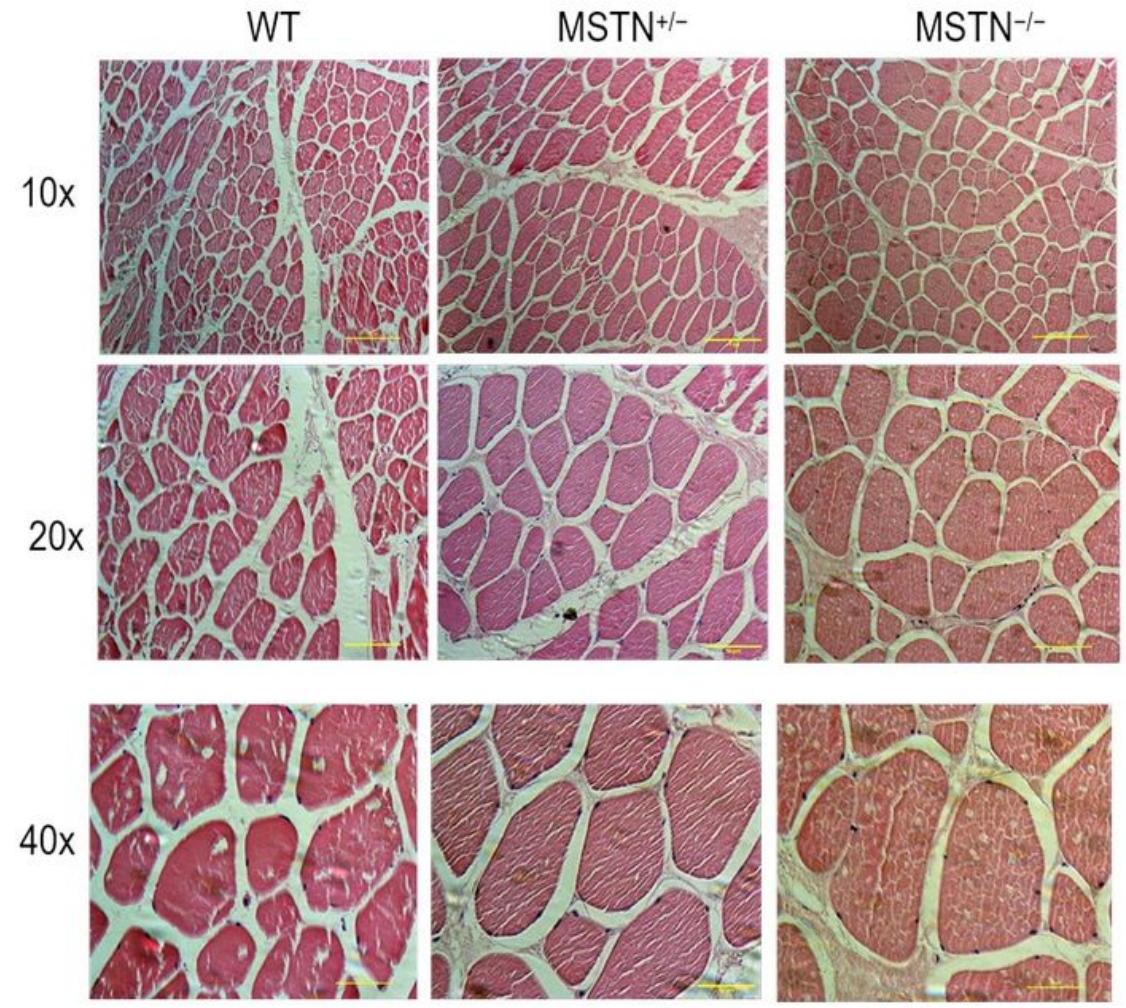

C

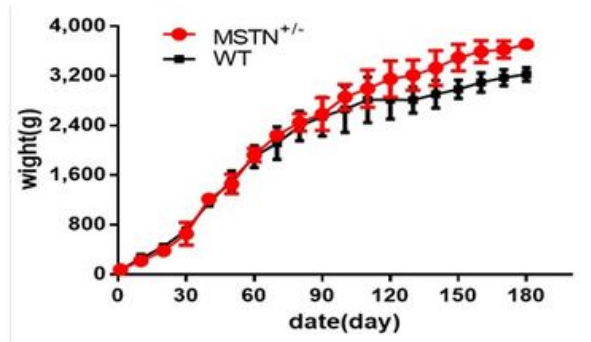

\section{Figure 7}

Detection and observation of MSTN-KO rabbits. (A) Comparison of mediated and wild type Rabbits at 3 and 6 months of age. (B) H\&E staining of the muscle fibers from gluteus maximus. (C) The average body weight of MSTN+/- and WT rabbits from FO $(n=5)$. 
A

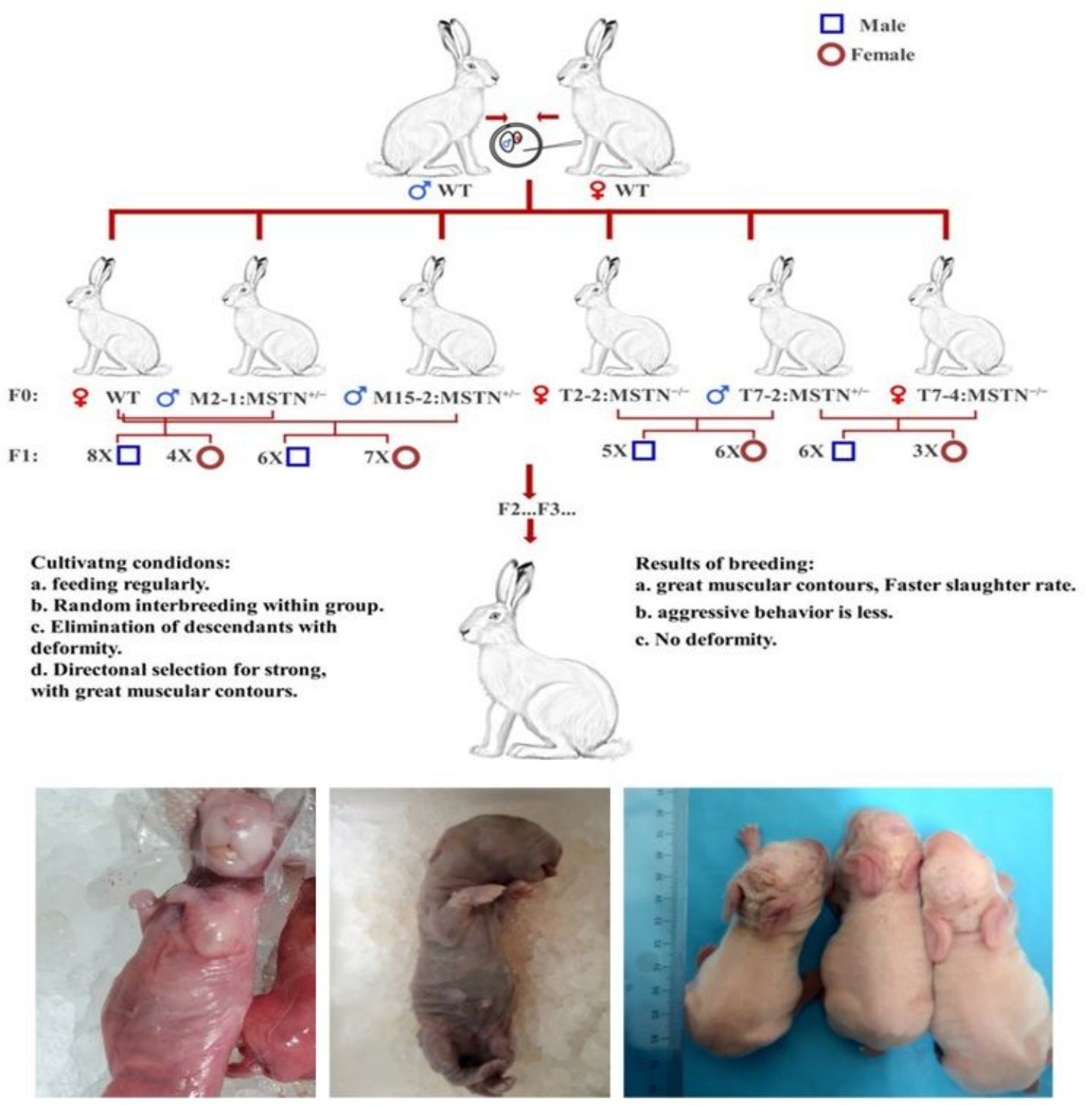

C

WT I GGGATtGGATTATTGCGCCCAAGAGATACAAGGCCAATTACT // TTGTACACCAAGCAAACCCCAGAGGTTCAGC 0

1 GGGATTGGATTATTGCGCCCAAGAGATACAAGGCCAATTACT // TTGTACACCAAGCAAACCCCAGAGGTTCAGC 0 GGGATTGGATTATTGCGCCCAAGAGATTACAAGGCCAATTACT // TTGTACACCAAGCAAACCCCAGAGGTTCAGC +1(T)

2 GGGATTGGATTATTGCGCCCAAGAGATACAAGGCCAATTACT // TTGTACACCAAGCAAACCCCAGAGGTTCAGC 0 GGGATTGGATTATTGCGCCCAAG …-_..-GCCAATTACT // TTGTACACCAAGCAAACCCCAGAGGTTCAGC

\section{Figure 8}

(A) Schematic diagram of MSTN KO Rabbits breeding family. (B) Left: Pups with big tongue. Right: Dystocia due to the large size of the pups. (C) Rabbits of F1 were named on the left. 1-2 represent different rabbit, PAM sequence is marked in green. The sgRNAs sequence is marked in red. The numbers on the right indicated the type of mutation, with "-" represents deletion of the given number of nucleotides, " + " represents insertion ofthe given number of nucleotides. 
A

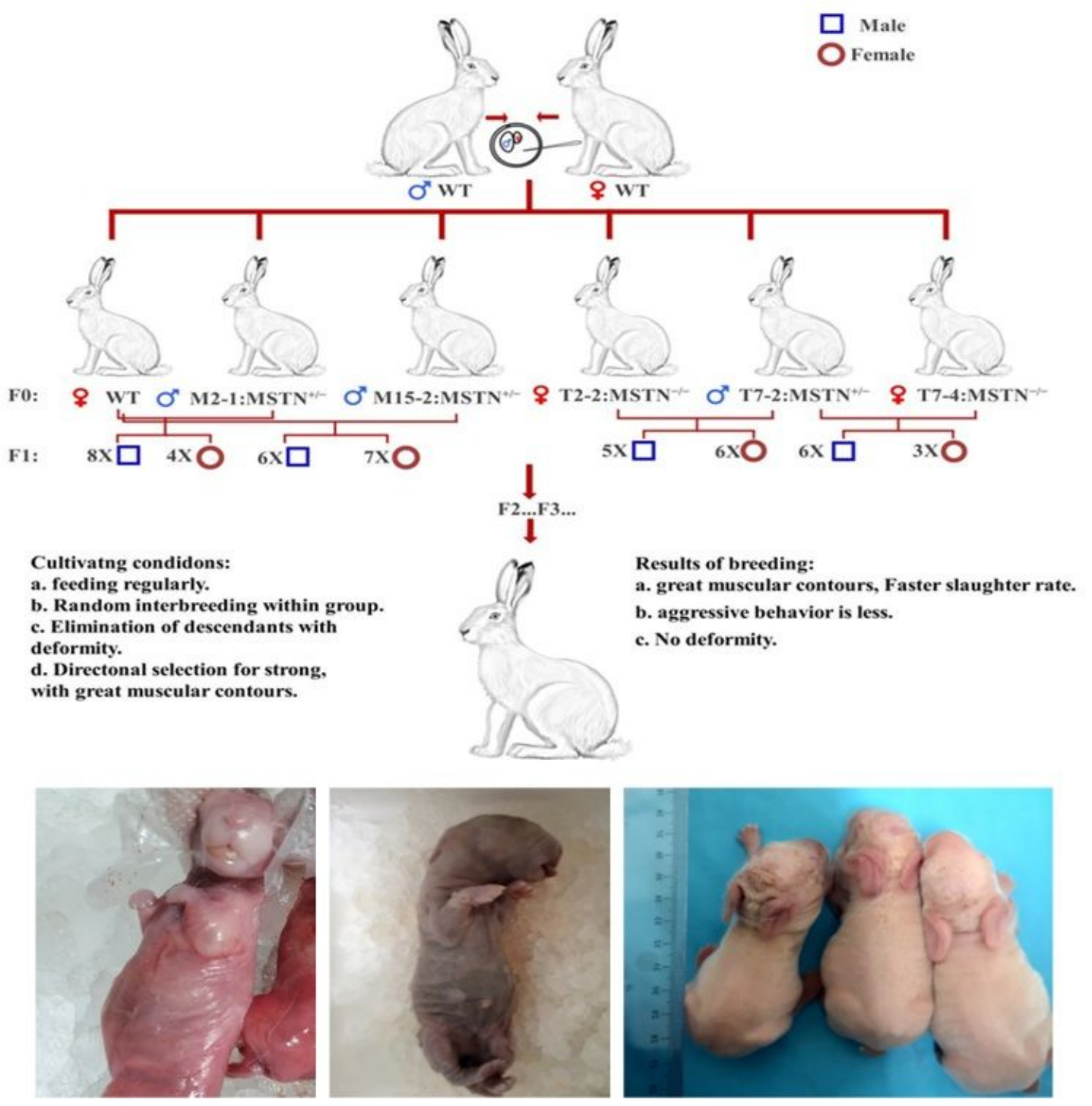

C

WT I GGGATtGGATTATTGCGCCCAAGAGATACAAGGCCAATTACT // TTGTACACCAAGCAAACCCCAGAGGTTCAGC 0

1 GGGATTGGATTATTGCGCCCAAGAGATACAAGGCCAATTACT // TTGTACACCAAGCAAACCCCAGAGGTTCAGC 0 GGGATTGGATTATTGCGCCCAAGAGATTACAAGGCCAATTACT // TTGTACACCAAGCAAACCCCAGAGGTTCAGC +1(T)

2 GGGATTGGATTATTGCGCCCAAGAGATACAAGGCCAATTACT // TTGTACACCAAGCAAACCCCAGAGGTTCAGC 0 GGGATTGGATTATTGCGCCCAAG …-_..-GCCAATTACT // TTGTACACCAAGCAAACCCCAGAGGTTCAGC

\section{Figure 8}

(A) Schematic diagram of MSTN KO Rabbits breeding family. (B) Left: Pups with big tongue. Right: Dystocia due to the large size of the pups. (C) Rabbits of F1 were named on the left. 1-2 represent different rabbit, PAM sequence is marked in green. The sgRNAs sequence is marked in red. The numbers on the right indicated the type of mutation, with "-" represents deletion of the given number of nucleotides, " + " represents insertion ofthe given number of nucleotides. 